\title{
Respiratory adverse effects of opioids for breathlessness: a systematic review and meta-analysis
}

\author{
Cindy A. Verberkt (10 ${ }^{1}$, Marieke H.J. van den Beuken-van Everdingen², \\ Jos M.G.A. Schols ${ }^{1,3}$, Sushma Datla ${ }^{4}$, Carmen D. Dirksen ${ }^{5}$, Miriam J. Johnson ${ }^{4}$, \\ Sander M.J. van Kuijk ${ }^{5}$, Emiel F.M. Wouters ${ }^{6,7}$ and Daisy J.A. Janssen ${ }^{2,6}$ \\ Affiliations: ${ }^{1}$ Dept of Health Services Research, Maastricht University, Maastricht, The Netherlands. ${ }^{2}$ Centre \\ of Expertise for Palliative Care, Maastricht University Medical Centre (MUMC+), Maastricht, The Netherlands. \\ ${ }^{3}$ Dept of Family Medicine, Maastricht University, Maastricht, The Netherlands. ${ }^{4}$ Hull York Medical School, \\ University of Hull, Hull, UK. ${ }^{5}$ Dept of Clinical Epidemiology and Medical Technology Assessment, Maastricht \\ University Medical Centre (MUMC+), Maastricht, The Netherlands. ${ }^{6} \mathrm{CIRO}$, Centre of expertise for chronic \\ organ failure, Horn, The Netherlands. ${ }^{7}$ Dept of Respiratory Medicine, Maastricht University Medical Centre \\ (MUMC+), Maastricht, The Netherlands.
}

Correspondence: C.A. Verberkt, P.O. Box 6166200 MD Maastricht, The Netherlands. E-mail: c.vandenbergla maastrichtuniversity.nl

@ERSpublications

There is no evidence of clinically relevant respiratory adverse effects of opioids for chronic breathlessness http://ow.ly/J61730eQNCR

Cite this article as: Verberkt CA, van den Beuken-van Everdingen MHJ,

Schols JMGA, et al. Respiratory adverse effects of opioids for breathlessness: a systematic review and metaanalysis. Eur Respir J 2017; 50: 1701153 [https://doi.org/10.1183/13993003.01153-2017].

ABSTRACT Previous studies have shown that opioids can reduce chronic breathlessness in advanced disease. However, physicians remain reluctant to prescribe opioids for these patients, commonly due to fear of respiratory adverse effects. The aim of this study was to systematically review reported respiratory adverse effects of opioids in patients with advanced disease and chronic breathlessness.

PubMed, Embase, the Cochrane Central Register of Controlled Trials, CINAHL, ClinicalTrials.gov and the reference lists of relevant systematic reviews were searched. Two independent researchers screened against predefined inclusion criteria and extracted data. Meta-analysis was conducted where possible.

We included 63 out of 1990 articles, describing 67 studies. Meta-analysis showed an increase in carbon dioxide tension $(0.27 \mathrm{kPa}, 95 \%$ CI $0.08-0.45 \mathrm{kPa}$ ) and no significant change in oxygen tension and oxygen saturation (both $\mathrm{p}>0.05$ ). Nonserious respiratory depression (definition variable/not stated) was described in four out of 1064 patients. One cancer patient pretreated with morphine for pain needed temporary respiratory support following nebulised morphine for breathlessness (single case study).

We found no evidence of significant or clinically relevant respiratory adverse effects of opioids for chronic breathlessness. Heterogeneity of design and study population, and low study quality are limitations. Larger studies designed to detect respiratory adverse effects are needed.

This article has supplementary material available from erj.ersjournals.com

Received: March 302017 | Accepted after revision: Aug 182017

Support statement. This project was funded by the Netherlands Organisation for Health Research and Development (ZonMW) (grant number 836031012). Funding information for this article has been deposited with the Crossref Funder Registry.

Conflict of interest: Disclosures can be found alongside this article at erj.ersjournals.com

Copyright @ERS 2017 


\section{Introduction}

Breathlessness is defined as "a subjective experience of breathing discomfort that consists of qualitatively distinct sensations that vary in intensity" [1]. Breathlessness is one of the most uncomfortable symptoms in patients with advanced disease [1]. In cancer, $50-70 \%$ of patients suffer from breathlessness, while in chronic obstructive pulmonary disease (COPD) this prevalence is as much as $56-98 \%[2,3]$.

Opioids can reduce chronic breathlessness (breathlessness that persists despite optimal treatment of the underlying pathophysiology and results in disability [4]) in patients with advanced diseases [5-8]. However, while physicians are mostly willing to prescribe opioids for breathlessness in the last days or weeks of life, they are often reluctant to prescribe opioids to those earlier in their disease trajectory [9]. Their main concerns are fears of respiratory adverse effects and lack of evidence-based guidelines [10-12]. Data about respiratory adverse effects of opioids are limited and conflicting. Systematic reviews on the effects of opioids on chronic breathlessness in adults with advanced life-limiting disease showed no evidence for the following outcomes: respiratory depression, increase in arterial carbon dioxide tension $\left(P_{\mathrm{aCO}}\right)$, increase in end-tidal carbon dioxide tension $\left(P \mathrm{ETCO}_{2}\right)$, decrease in arterial oxygen tension $\left(\mathrm{PaO}_{2}\right)$ or decrease in arterial oxygen saturation $\left(\mathrm{SaO}_{2}\right)$ [5-8]. However, meta-analyses on these outcomes have not been conducted before.

Conversely, observational studies have reported one or more cases of severe respiratory depression in patients using opioids for breathlessness [13-16]. Most guidelines in palliative care recommend the use of opioids for chronic breathlessness [17-19]. However, guidelines in respiratory medicine, for example the recent Global Initiative for Chronic Obstructive Lung Disease guidelines [20], are more circumspect because of possible serious adverse events and limited effectiveness. To date there is little evidence whether and to what extent opioids lead to respiratory adverse effects in patients with chronic breathlessness.

The aim of this systematic review and meta-analysis was to study the occurrence of respiratory adverse effects (in particular increase of $\mathrm{PaCO}_{2}$ and $\mathrm{PETCO}_{2}$, decrease of $\mathrm{PaO}_{2}$ and $\mathrm{SaO}_{2}$, decrease in respiratory rate, and occurrence of respiratory depression) in patients with advanced disease and chronic breathlessness who are treated with opioids. Respiratory adverse effects are examined in experimental studies and observational studies, as well as case reports. However, none of the previous reported reviews included all these study types. Therefore, to generate a full overview of the current knowledge, we included experimental studies, observational studies and case reports.

\section{Methods}

A systematic review and meta-analysis was performed according to the Cochrane methodology [21]. Results are reported in accordance with the Preferred Reporting Items for Systematic Reviews and Meta-Analyses (PRISMA) statement [22]. The protocol is registered in the International Prospective Register of Systematic Reviews (PROSPERO; CRD42016033691).

\section{Search strategy}

The following databases were searched: PubMed, Embase on Ovid, Cochrane Central Register of Controlled Trials and CINAHL on EBSCO (inception date to March 31, 2016). Search terms comprised (dyspnoea OR synonyms) AND (opioid OR synonyms) and included both terms of controlled vocabulary and free search in title and abstract (online supplementary tables S1a-S1d). Furthermore, ClinicalTrials.gov was searched for ongoing or completed studies using the same search terms (May 29, 2017; online supplementary table S1e). Following de-duplication, we included all original research articles such as randomised controlled trials (RCTs), nonrandomised trials, case-control studies, cohort studies, chart reviews, case reports and case studies. Reference lists of three relevant systematic reviews [6-8] were searched by hand and experts in the field were contacted. We included articles in the English, Dutch, German, French and Spanish languages. When a full-text article was not accessible, this was requested from the authors.

\section{Study selection}

For study screening, we used Endnote X7 (Thomson Reuters, Philadelphia, PA, USA). The titles and abstracts were screened independently by two researchers (CV and either DJ, MvdB or SD) and selected based on the description of treatment for chronic breathlessness using opioids. The remaining full-text articles were screened by two researchers (CV and either SD (English), DJ (German or Dutch) or LV (French or Spanish)) against all eligibility criteria: 1) participants included patients, regardless of their primary condition; 2) any opioid as intervention prescribed for breathlessness, regardless of dose or route of prescription; and 3) primary or secondary outcomes included $\mathrm{PaCO}_{2}, \mathrm{PaO}_{2}, \mathrm{SaO}_{2}$ or respiratory rate. During the screening process, we decided in addition to include $\mathrm{PETCO}_{2}$, occurrence of respiratory depression and breathlessness as outcomes. Any type of control group was considered. We excluded 
studies including only healthy subjects or studies that used an opioid in combination with other treatments and the effect of the opioid could not be distinguished. Consensus was reached by discussion. The study designs of included articles were categorised as follows: RCTs, nonrandomised trials (NRTs), prospective observational studies (POSs), retrospective observational studies (ROSs) and case reports.

\section{Risk of bias}

Two researchers independently assessed the risk of bias on the study level (CV and either SD (English), DJ (German) or LV (French)). For the RCTs, we assessed this risk of bias regarding random sequence generation, allocation concealment, blinding, incomplete outcome data and selective reporting using the Cochrane risk of bias tool [21]. In addition, the Cochrane risk of bias tool was used to assess the risk of bias in NRTs. Since no control condition was included in these studies, a high risk of selection bias, performance bias and detection bias were estimated in all NRTs. For POSs, we assessed the risk of bias regarding selection, comparability and exposure/outcome using the Newcastle-Ottawa quality assessment scale [23]. Consensus was reached by discussion. The risk of bias in ROSs and case reports was not assessed.

\section{Data collection}

Data were extracted by two researchers (CV and either SD (English), DJ (German) or LV (French)) using a predefined extraction form in Excel (Microsoft, Redmond, WA, USA), including data on study characteristics (design, duration, setting and inclusion and exclusion criteria); type of intervention (intervention, comparison, dose, mode and timing of administration); study population (sample size, age, sex, diagnosis, disease severity and use of oxygen); and outcomes (breathlessness; respiratory outcomes: $\mathrm{PaCO}_{2}, \mathrm{PETCO}_{2}, \mathrm{PaO}_{2}, \mathrm{SaO}_{2}$, respiratory rate and occurrence of respiratory depression; mode of assessment; and missing data). When two articles appeared to describe overlapping research questions and study populations, we contacted the authors to request more information. We recorded the baseline values and change from baseline or post-treatment scores of the respiratory outcomes. When only a description of the change from baseline was given, this was taken into account. The form was piloted on two articles of each study type and adapted as needed.

\section{Data synthesis}

Change from baseline measurement scores or post-treatment measurement scores were collected for the $\mathrm{PaCO}_{2}, \mathrm{PETCO}_{2}, \mathrm{PaO}_{2}, \mathrm{SaO}_{2}$ and respiratory rate. For the RCTs, these results were compared between the intervention and control groups. For the NRTs, POSs, ROSs and case reports, the change from baseline was examined. Meta-analyses were performed using the results of RCTs; however, RCTs without a placebo comparator group were not included. When both a change from baseline and a post-treatment score were reported, the post-treatment score was used in the meta-analyses. Furthermore, the highest dose or latest measurement was included in the meta-analyses if multiple doses of the same opioid or repeated measurements were reported. When an RCT compared more than one opioid with placebo, the morphine group was included in the meta-analysis. For measurements on exertion, the submaximal measures at a fixed time point were included. To verify whether the included RCTs showed a pooled effect of improving breathlessness, meta-analysis on the effect of opioids on breathlessness was performed. These results were presented as standardised mean difference $(95 \% \mathrm{CI})$, since different scales to measure breathlessness were used. Results of the meta-analyses on $\mathrm{PaCO}_{2}, \mathrm{EtCO}_{2}, \mathrm{PaO}_{2}, \mathrm{SaO}_{2}$ and respiratory rate were presented as mean difference (MD) (95\% CI), as the same scales to measure comparable outcomes were used. In all meta-analyses a random effects model was used, since the study designs were heterogeneous [21]. Results of $\mathrm{PaO}_{2}$ and $\mathrm{PaCO}_{2}$ that were reported in $\mathrm{mmHg}$ were converted to $\mathrm{kPa}(1 \mathrm{mmHg}=0.133 \mathrm{kPa})$.

Some RCTs contributed more than one contrast between the opioid and control group for the same outcome (i.e. subjects were measured multiple times under comparable conditions). To account for this clustering of multiple contrasts within one study sample, we used a multilevel meta-analysis approach to determine if any within-study clustering was present. If there was evidence of within-study clustering, quantified by the intraclass correlation coefficient, the results of the multilevel approach were preferred over the standard approach [24]. To examine the impact of the context of assessment (at rest or on exertion), the number of doses (single dose or multiple doses) or the route of administration (nebulised or systemic), a mixed-effects meta-regression was performed. Subgroup analyses were performed for variables which appeared to be of impact. When no impact appeared, all outcomes were analysed together.

When a study assessed the occurrence of respiratory depression, the frequency of occurrence and the definition used was reported. Analysis of this outcome was descriptive.

Analyses were performed using Review Manager (version 5.3; The Northern Cochrane Centre, 2014, Copenhagen, Denmark) and R (version 3.2.3; R Foundation for Statistical Computing, Vienna, Austria). 
GRADEPro Guideline Development Tool software (www.gradepro.org) was used to construct the summary of findings table. Results are shown per category of respiratory adverse effect. p-values $\leqslant 0.05$ were considered statistically significant.

\section{Results}

\section{Study characteristics}

The search identified 1990 articles, of which 63 met the inclusion criteria (figure 1). The 63 articles included reported on 67 studies: 35 RCTs (table 1), 17 NRTs (table 1), four POSs (online supplementary table S2), five ROSs (online supplementary table S2) and six case reports (online supplementary table S3). Six ongoing studies, four RCTs and two NRTs, were identified (online supplementary table S4). $P_{\mathrm{aCO}_{2}}$, $\mathrm{PaO}_{2}$ and $\mathrm{PETCO}_{2}$ are examined in one study; $\mathrm{SaO}_{2}$ is examined in four studies and respiratory rate is examined in three studies. In one study, it is not clear which blood gases are examined. In one study the respiratory adverse effects are a primary outcome, and in five studies the respiratory adverse effects are secondary outcomes.

19 RCTs were included in the meta-analysis on the effect of opioid treatment on breathlessness [25-42]. Eight RCTs used a visual analogue scale to examine breathlessness [25, 26, 29, 35, 36, 38, 40]; six RCTs used the Borg scale [27, 30-34]; three RCTs used a numeric rating scale [28, 39, 41]; one RCT used the dyspnoea domain of the Chronic Respiratory Questionnaire [42] and one RCT used an oxygen cost diagram [37]. The RCTs that reported post-treatment scores showed effectivity of opioids in relieving breathlessness (standardised mean difference $-0.42,95 \%$ CI $-0.62--0.21$; I $27 \%$; online supplementary figure S1). The RCTs that reported changes from baseline were not able to show effectivity of opioids in relieving breathlessness (standardised mean difference -0.09 , 95\% CI $-0.78-0.60 ; \mathrm{I}^{2} 62 \%$; online supplementary figure S1).

\section{Risk of bias}

As shown in online supplementary table S5, the risk of bias of the RCTs was estimated to be low or unclear in most studies. Other sources of bias were assessed as high risk in $43 \%$ of the studies, mainly because of the absence of a washout period in crossover trials. Online supplementary table S6 shows the

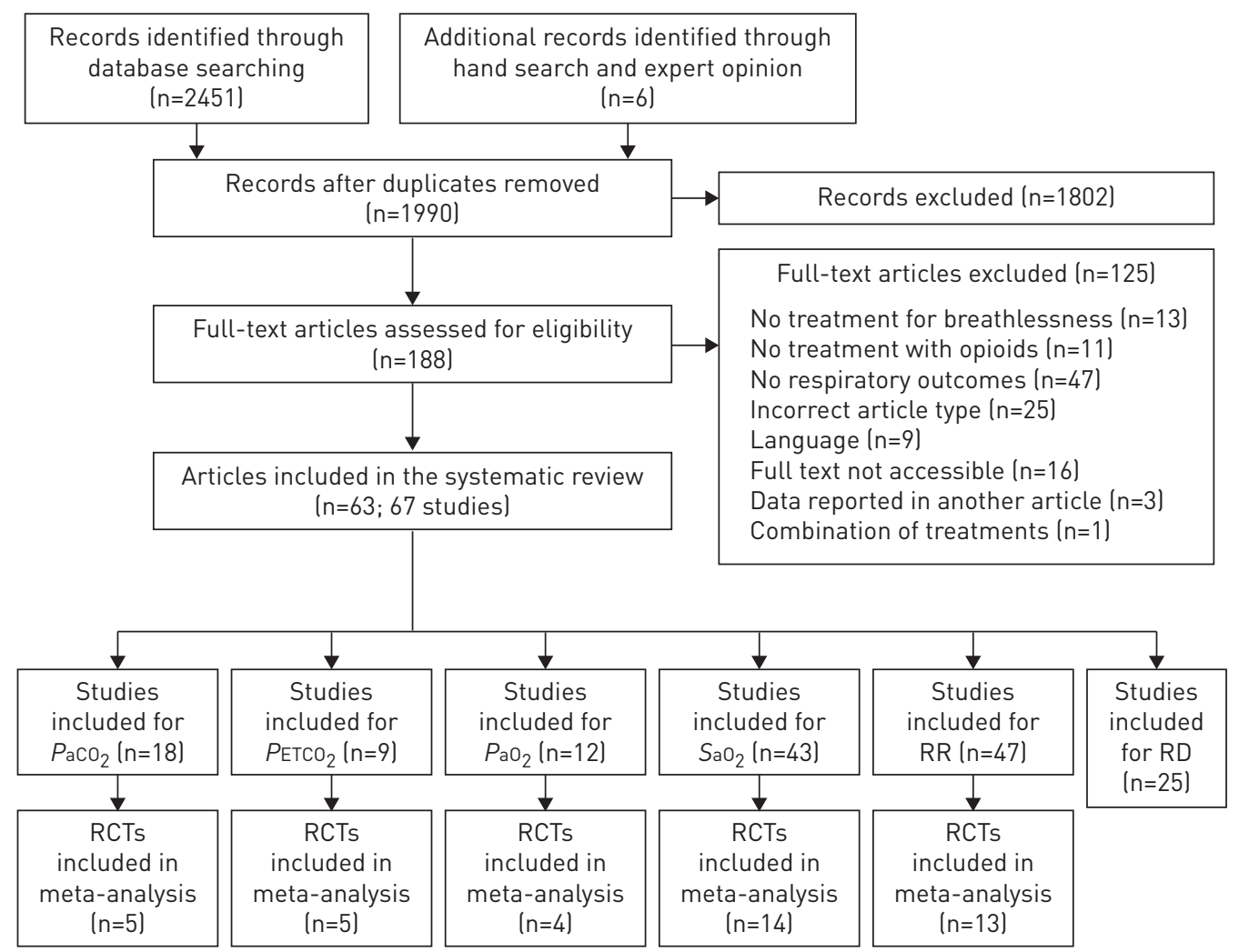

FIGURE 1 Study selection. $\mathrm{PaCO}_{2}$ : arterial carbon dioxide tension; $\mathrm{PETCO}_{2}$ : end-tidal carbon dioxide tension; $\mathrm{PaO}_{2}$ : arterial oxygen tension; $\mathrm{SaO}_{2}$ : arterial oxygen saturation; RR: respiratory rate; RD: respiratory depressions; RCTs: randomised controlled trials. 
TABLE 1 Patient characteristics, study design and included outcomes of randomised controlled trials and nonrandomised trials

\begin{tabular}{|c|c|c|c|c|c|c|c|c|c|c|c|}
\hline $\begin{array}{l}\text { First author, } \\
\text { year } \\
\text { [reference] }\end{array}$ & Design & $\begin{array}{c}\text { Subjects } \\
\text { n (\% } \\
\text { male) }\end{array}$ & $\begin{array}{l}\text { Population } \\
\text { (n) }\end{array}$ & Age years & Opioid & Dose & Administration & Comparison & Duration & $\begin{array}{l}\text { Patient } \\
\text { setting }\end{array}$ & $\begin{array}{l}\text { Included } \\
\text { outcomes }\end{array}$ \\
\hline $\begin{array}{l}\text { ABERNETHY, } \\
2003[25]\end{array}$ & Crossover & 48 (73) & $\begin{array}{l}\text { COPD (42) } \\
\text { Cancer (3) } \\
\text { MND (1) } \\
\text { RLD (2) }\end{array}$ & $76 \pm 5$ & Morphine SR & $20 \mathrm{mg} \cdot \mathrm{day}^{-1}$ & Oral & Placebo & 4 days & Outpatient & $\begin{array}{l}\mathrm{SaO}_{2}, \mathrm{RR} \\
\mathrm{RD}\end{array}$ \\
\hline $\begin{array}{l}\text { AlLARD, } 1999 \\
\text { [43] }\end{array}$ & Parallel & $33(42)$ & Cancer (33) & 63.3 & $\begin{array}{l}\text { Based on current } \\
\text { treatment }\end{array}$ & $50 \%$ of current dose ${ }^{\#}$ & $\begin{array}{l}\text { Oral or } \\
\text { parenteral }\end{array}$ & $\begin{array}{c}25 \% \text { of current } \\
\text { dose }^{\#}\end{array}$ & $\begin{array}{l}\text { Single } \\
\text { dose }\end{array}$ & Inpatient & $\mathrm{RR}$ \\
\hline $\begin{array}{l}\text { BeAuford, } 1993 \\
\text { [44] }\end{array}$ & Crossover & $8(88)$ & COPD (8) & $60.8 \pm 9.1$ & Morphine & 1,4 or $10 \mathrm{mg}$ & Nebulised & Placebo & $\begin{array}{l}\text { Single } \\
\text { dose }\end{array}$ & Outpatient & $P_{\mathrm{ETCO}}$ \\
\hline $\begin{array}{l}\text { BRUERA, } 1993 \\
\text { (part 1) } \\
\text { [26] }\end{array}$ & Crossover & 10 & Cancer (10) & No data & Morphine & $\begin{array}{l}\text { Target: } 150 \% \text { of } \\
\text { current dose (34 } \\
\quad \pm 12 \mathrm{mg} \text { ) }\end{array}$ & Parenteral & Placebo & $\begin{array}{l}\text { Single } \\
\text { dose }\end{array}$ & Inpatient & $\mathrm{SaO}_{2}, \mathrm{RR}$ \\
\hline $\begin{array}{l}\text { ChARLES, } 2008 \\
\text { [38] }\end{array}$ & Crossover & 20 (55) & Cancer (20) & $\begin{array}{c}69 \text { (range 48- } \\
83 \text { ) }\end{array}$ & Hydromorphone $e^{\#, q}$ & $5 \mathrm{mg}$ & Nebulised & Placebo" & $\begin{array}{l}\text { Single } \\
\text { dose }\end{array}$ & $\begin{array}{l}\text { Inpatient/ } \\
\text { outpatient }\end{array}$ & $\mathrm{SaO}_{2}, \mathrm{RR}$ \\
\hline $\begin{array}{c}\text { CHUA, } 1997 \\
\text { [27] }\end{array}$ & Crossover & $12(100)$ & $\mathrm{CHF}$ (12) & $65.5 \pm 1.5$ & Dihydrocodeine & $\begin{array}{c}1 \mathrm{mg} \cdot \mathrm{kg}^{-1} \text { body } \\
\text { weight }(77.4 \pm 3.1 \mathrm{~kg})\end{array}$ & Oral & Placebo & $\begin{array}{l}\text { Single } \\
\text { dose }\end{array}$ & Unclear & $\begin{array}{l}P_{\mathrm{ETCO}_{2}} \\
\mathrm{SaO}_{2}, \mathrm{RR}\end{array}$ \\
\hline $\begin{array}{c}\text { Cuervo Pinna, } \\
2015 \text { [28] }\end{array}$ & Crossover & $13(85)$ & Cancer (13) & $65.2 \pm 10.4$ & Fentanyl & $\begin{array}{l}\text { Opioid-naïve: } 200 \mu \mathrm{g} \\
\text { Pretreated: } 400 \mu \mathrm{g}\end{array}$ & Oral & Placebo & $\begin{array}{l}\text { Single } \\
\text { dose }\end{array}$ & Unclear & $\mathrm{SaO}_{2}, \mathrm{RR}$ \\
\hline $\begin{array}{l}\text { EISER, } 1991 \\
\text { (part 1) } \\
\text { [29] }\end{array}$ & Crossover & $14(57)$ & COPD (14) & $\begin{array}{c}65 \text { (range 49- } \\
79 \text { ) }\end{array}$ & Diamorphine & 10 or $20 \mathrm{mg} \cdot \mathrm{day}^{-1}$ & Oral & Placebo & 2 weeks & Outpatient & $\begin{array}{c}\mathrm{PaCO}_{2} \\
\mathrm{PETCO}_{2} \\
\mathrm{PaO}_{2}, \mathrm{SaO}_{2}\end{array}$ \\
\hline $\begin{array}{l}\text { EISER, } 1991 \\
\text { (part 2) } \\
\text { [29] }\end{array}$ & Crossover & $10(60)$ & COPD (10) & $\begin{array}{c}65 \text { (range 49- } \\
79 \text { ) }\end{array}$ & Diamorphine & $15 \mathrm{mg} \cdot \mathrm{day}^{-1}$ & Oral & Placebo & 1 day & Outpatient & $\mathrm{PaCO}_{2}, \mathrm{PaO}_{2}$ \\
\hline $\begin{array}{l}\text { GaMBORG, } 2013 \\
\text { [45] }\end{array}$ & Parallel & $20(10)$ & Cancer (20) & $\begin{array}{c}\text { Median } 69 \\
\text { (range 42-84) }\end{array}$ & Morphine & $\begin{array}{l}\text { Target: } 1 / 12 \text { of total } \\
\text { daily dose with a } \\
\text { maximum of } 24 \mathrm{mg} \text { ) } \\
\text { (median } 8.2 \% \text { ) }\end{array}$ & Oral & $\begin{array}{l}\text { Subcutaneous } \\
\text { morphine; } 60 \% \text { of } \\
1 / 12 \text { of total daily } \\
\text { dose with a } \\
\text { maximum of } \\
14.4 \mathrm{mg}\end{array}$ & $\begin{array}{l}\text { Single } \\
\text { dose }\end{array}$ & Inpatient & $\begin{array}{l}\mathrm{SaO}_{2}, \mathrm{RR} \\
\mathrm{RD}\end{array}$ \\
\hline $\begin{array}{l}\text { GRIMBERT, } 2004 \\
\text { [46] }\end{array}$ & Crossover & 12 (92) & Cancer (12) & $\begin{array}{c}63 \text { (range } 44- \\
82 \text { ) }\end{array}$ & Morphine & $120 \mathrm{mg} \cdot \mathrm{day}^{-1}$ & Nebulised & Placebo & 2 days & Inpatient & $\mathrm{SaO}_{2}, \mathrm{RR}$ \\
\hline $\begin{array}{l}\text { HARRIS-EZE, } \\
1995 \text { [30] }\end{array}$ & Crossover & $6(83)$ & ILD (6) & $49 \pm 16$ & Morphine & $\begin{array}{c}\text { Target: } 2.5 \mathrm{mg} \text { (mean } \\
1.9 \mathrm{mg} \text { or } 5 \mathrm{mg} \\
\text { (mean } 3.7 \mathrm{mg} \text { ) }\end{array}$ & Nebulised & Placebo & $\begin{array}{l}\text { Single } \\
\text { dose }\end{array}$ & Outpatient & $\begin{array}{c}\mathrm{PETCO}_{2} \\
\mathrm{SaO}_{2}\end{array}$ \\
\hline Hul, 2014 [39] & Parallel & $20(45)$ & Cancer (20) & $\begin{array}{c}55 \text { (range 27- } \\
75 \text { ) }\end{array}$ & Fentanyl $^{+}$ & $30-350 \mu \mathrm{g}^{\S}$ & Parenteral & Placebo & $\begin{array}{l}\text { Single } \\
\text { dose }\end{array}$ & Outpatient & $\mathrm{SaO}_{2}, \mathrm{RR}$ \\
\hline $\begin{array}{l}\text { JANKELSON, } \\
1997[31]\end{array}$ & Crossover & $16(69)$ & COPD (16) & 69 (range 61- & Morphine & 20 or $40 \mathrm{mg}$ & Nebulised & Placebo & $\begin{array}{l}\text { Single } \\
\text { dose }\end{array}$ & Inpatient & $\mathrm{SaO}_{2}$ \\
\hline $\begin{array}{l}\text { JENSEN, } 2012 \\
\text { [32] }\end{array}$ & Crossover & $16(58)$ & COPD (16) & $70.5 \pm 2.3$ & Fentanyl & $50 \mu \mathrm{g}$ & Nebulised & Placebo & $\begin{array}{l}\text { Single } \\
\text { dose }\end{array}$ & Unclear & $\begin{array}{l}P \mathrm{ETCO}_{2} \\
\mathrm{SaO}_{2}, \mathrm{RR} \\
\mathrm{RD}\end{array}$ \\
\hline
\end{tabular}




\begin{tabular}{|c|c|c|c|c|c|c|c|c|c|c|c|}
\hline $\begin{array}{l}\text { First author, } \\
\text { year } \\
\text { [reference] }\end{array}$ & Design & $\begin{array}{c}\text { Subjects } \\
\text { n (\% } \\
\text { male) }\end{array}$ & $\begin{array}{l}\text { Population } \\
\text { (n) }\end{array}$ & Age years & Opioid & Dose & Administration & Comparison & Duration & $\begin{array}{l}\text { Patient } \\
\text { setting }\end{array}$ & $\begin{array}{l}\text { Included } \\
\text { outcomes }\end{array}$ \\
\hline $\begin{array}{l}\text { Johnson, } 2002 \\
\text { [47] }\end{array}$ & Crossover & $10(100)$ & $\mathrm{CHF}(10)$ & $\begin{array}{l}67 \text { (range } \\
45-85 \text { ) }\end{array}$ & Morphine & $10-20 \mathrm{mg} \cdot \mathrm{day}^{-1}$ & Oral & Placebo & 4 days & Inpatient & $\begin{array}{c}\mathrm{PaCO}_{2}, \\
\mathrm{PaO}_{2}, \mathrm{SaO}_{2,} \\
\mathrm{RR}\end{array}$ \\
\hline $\begin{array}{l}\text { KRAJNIK, } 2009 \\
\text { [48] }\end{array}$ & Parallel & $10(40)$ & Cancer (10) & $\begin{array}{c}55.5 \text { (range } \\
39-73 \text { ) }\end{array}$ & Morphine & $5 \mathrm{mg}$ & Nebulised & $\begin{array}{l}2 \text { types of } \\
\text { nebulisation }\end{array}$ & $\begin{array}{l}\text { Single } \\
\text { dose }\end{array}$ & Inpatient & $\begin{array}{c}\mathrm{PaCO}_{2} \\
\mathrm{PaO}_{2}, \mathrm{SaO}_{2}\end{array}$ \\
\hline $\begin{array}{l}\text { LIGHT, } 1989 \\
\text { [33] }\end{array}$ & Crossover & $13(100)$ & COPD (13) & $\begin{array}{l}65.9 \text { (range } \\
58-70 \text { ) }\end{array}$ & Morphine & $0.8 \mathrm{mg} \cdot \mathrm{kg}^{-1}$ & Oral & Placebo & $\begin{array}{l}\text { Single } \\
\text { dose }\end{array}$ & Unclear & $\begin{array}{c}\mathrm{PaCO}_{21} \\
\mathrm{PaO}_{2}, \mathrm{SaO}_{2,} \\
\mathrm{RR}\end{array}$ \\
\hline $\begin{array}{l}\text { LIGHT, } 1996 \\
\text { [34] }\end{array}$ & Crossover & $7(100)$ & COPD (7) & $66.4 \pm 3.3$ & Morphine & $30 \mathrm{mg}$ & Oral & Placebo & $\begin{array}{l}\text { Single } \\
\text { dose }\end{array}$ & Unclear & $P \mathrm{ETCO}_{2}$ \\
\hline $\begin{array}{l}\text { Masood, } 1995 \\
\text { [49] }\end{array}$ & Crossover & $12(100)$ & COPD (12) & $66.3 \pm 7.0$ & Morphine & $\begin{array}{c}\text { Nebulised: } 10 \text { and } \\
25 \mathrm{mg} \\
\text { Parenteral: } 1 \text { and } \\
2.5 \mathrm{mg}\end{array}$ & $\begin{array}{l}\text { Nebulised and } \\
\text { parenteral }\end{array}$ & Placebo & $\begin{array}{l}\text { Single } \\
\text { dose }\end{array}$ & Inpatient & $\mathrm{SaO}_{2}, \mathrm{RR}$ \\
\hline $\begin{array}{l}\text { Mazzocato, } \\
1999[40]\end{array}$ & Crossover & $9(66)$ & Cancer (9) & $\begin{array}{c}73 \text { (range } \\
66-83 \text { ) }\end{array}$ & Morphine & $\begin{array}{l}5 \mathrm{mg} \text { lor } 150 \% \text { of } \\
\text { pretreatment dose) }\end{array}$ & Parenteral & Placebo & $\begin{array}{l}\text { Single } \\
\text { dose }\end{array}$ & Inpatient & $\begin{array}{l}\mathrm{SaO}_{2}, \mathrm{RR} \\
\mathrm{RD}\end{array}$ \\
\hline $\begin{array}{l}\text { Munck, } 1990 \\
\text { (part 2) } \\
{[50]}\end{array}$ & Crossover & 21 & COPD (21) & $\begin{array}{c}\text { Median } 67 \\
\text { (range } 50-78 \text { ) }\end{array}$ & Codeine & $60 \mathrm{mg} \cdot \mathrm{day}^{-1}$ & Oral & $1 \mathrm{~g}$ paracetamol & 7 days & Inpatient & $\begin{array}{c}\mathrm{PaCO}_{2}, \\
\mathrm{PaO}_{2}, \mathrm{SaO}_{2,} \\
\mathrm{RR}\end{array}$ \\
\hline $\begin{array}{l}\text { Natalini, } 2011 \\
\text { [51] }\end{array}$ & Crossover & $13(43)$ & $\begin{array}{c}\text { Trauma (3) } \\
\text { COPD (3) } \\
\text { Pneumonia } \\
\text { (3) } \\
\text { Stroke (2) } \\
\text { Epilepsy (1) } \\
\text { Peritonitis (1) }\end{array}$ & $\begin{array}{l}\text { Median } 78 \\
\text { (IQR 73-82) }\end{array}$ & Remifentanyl & $0.05 \mu \mathrm{g} \cdot \mathrm{kg}^{-1} \cdot \mathrm{min}^{-1}$ & Parenteral & Placebo & $\begin{array}{l}\text { Single } \\
\text { dose }\end{array}$ & Inpatient & $\begin{array}{c}\mathrm{PaCO}_{2} \\
\mathrm{PaO}_{2}, \mathrm{RR}\end{array}$ \\
\hline $\begin{array}{l}\text { Navigante, } \\
2010 \text { [52] }\end{array}$ & Parallel & 63 & Cancer (63) & $\begin{array}{c}\text { Median } 55 \\
\text { (range } 30-80 \text { ) }\end{array}$ & Morphine ${ }^{\#}$ & $22.5(4.12) \mathrm{mg}$ & Oral & Midazolam & 5 days & Outpatient & $\mathrm{SaO}_{2}$ \\
\hline $\begin{array}{l}\text { Noseda, } 1997 \\
\text { [35] }\end{array}$ & Crossover & $17(76)$ & $\begin{array}{l}\text { COPD (12) } \\
\text { IPF (1) } \\
\text { Cancer (3) } \\
\text { CHF (1) }\end{array}$ & $69 \pm 11$ & Morphine & 10 or $20 \mathrm{mg}$ & Nebulised & Placebo & $\begin{array}{l}\text { Single } \\
\text { dose }\end{array}$ & Inpatient & $\begin{array}{l}\mathrm{SaO}_{2} \\
\mathrm{RR}\end{array}$ \\
\hline $\begin{array}{l}\text { Otulana, } 2004 \\
\text { (phase 3) } \\
\text { [53] }\end{array}$ & Crossover & 19 & Asthma (19) & Range 19-64 & Morphine & $2.2,4.4$ or $8.8 \mathrm{mg}$ & Nebulised & 3 doses & $\begin{array}{l}\text { Single } \\
\text { dose }\end{array}$ & Unclear & $\mathrm{RR}$ \\
\hline $\begin{array}{l}\text { OXBERRY, } 2011 \\
\text { [41] }\end{array}$ & Crossover & $35(86)$ & CHF (35) & $70.2 \pm 11.1$ & $\begin{array}{l}\text { Morphine } \\
\text { Oxycodone }\end{array}$ & $\begin{array}{l}20 \mathrm{mg} \cdot \mathrm{day}^{-1} \\
10 \mathrm{mg} \cdot \mathrm{day}^{-1}\end{array}$ & Oral & Placebo & 4 days & Outpatient & $\mathrm{SaO}_{2}, \mathrm{RR}$ \\
\hline $\begin{array}{l}\text { PoOLE, } 1998 \\
\text { [42] }\end{array}$ & Crossover & $16(69)$ & COPD (16) & $70.7 \pm 6.4$ & Morphine SR & $\begin{array}{l}\text { Target: } 40 \mathrm{mg} \\
\text { (mean } 25 \mathrm{mg} \text { ) }\end{array}$ & Oral & Placebo & 6 weeks & Outpatient & $\mathrm{SaO}_{2}$ \\
\hline
\end{tabular}




\begin{tabular}{|c|c|c|c|c|c|c|c|c|c|c|c|}
\hline $\begin{array}{l}\text { First author, } \\
\text { year } \\
\text { [reference] }\end{array}$ & Design & $\begin{array}{c}\text { Subjects } \\
\text { n (\% } \\
\text { male) }\end{array}$ & $\begin{array}{l}\text { Population } \\
\text { (n) }\end{array}$ & Age years & Opioid & Dose & Administration & Comparison & Duration & $\begin{array}{l}\text { Patient } \\
\text { setting }\end{array}$ & $\begin{array}{l}\text { Included } \\
\text { outcomes }\end{array}$ \\
\hline $\begin{array}{l}\text { RICE, } 1987 \\
\text { [54] }\end{array}$ & Crossover & 11 (100) & COPD (11) & Range 59-79 & Codeine & $120 \mathrm{mg}$ & Oral & Promethazine & 1 month & Unclear & $P \mathrm{aCO}_{2}, \mathrm{PaO}_{2}$ \\
\hline $\begin{array}{l}\text { RoBIN, } 1986 \\
\text { [55] }\end{array}$ & Crossover ${ }^{f}$ & $1(0)$ & OLD & 63 & Hydromorphone & $12 \mathrm{mg} \cdot \mathrm{day}^{-1}$ & Rectal & Placebo & $24 \mathrm{~h}$ & Outpatient & $\mathrm{PaCO}_{2}, \mathrm{PaO}_{2}$ \\
\hline $\begin{array}{l}\text { SCHONHOFER, } \\
1998[56]\end{array}$ & Crossover & $20(55)$ & $\begin{array}{l}\text { Lung } \\
\text { emphysema } \\
\text { (20) }\end{array}$ & $68.5 \pm 6.8$ & Morphine SR & $\begin{array}{l}\text { Target: } 90 \mathrm{mg} \\
\text { (mean } 49 \mathrm{mg} \text { ) }\end{array}$ & Oral & Usual care & 10 days & Inpatient & $\begin{array}{c}\mathrm{PaCO}_{2} \\
\mathrm{PaO}_{2}, \mathrm{RD}\end{array}$ \\
\hline $\begin{array}{l}\text { SHонRAтl, } 2012 \\
\text { [36] }\end{array}$ & Parallel & $40(100)$ & COPD (40) & No data & Morphine & $1 \mathrm{mg} \cdot \mathrm{day}^{-1}$ & Nebulised & Placebo & 5 days & Inpatient & $\mathrm{RR}$ \\
\hline $\begin{array}{l}\text { SMITH, } 2009 \\
\text { [57] }\end{array}$ & Crossover & $2(0)$ & $\begin{array}{l}\text { Cancer (1) } \\
\text { Unclear (1) }\end{array}$ & 49 and 59 & Fentanyl & $25 \mu \mathrm{g}$ & Nebulised & Placebo & $\begin{array}{l}\text { Single } \\
\text { dose }\end{array}$ & Inpatient & $\mathrm{SaO}_{2}, \mathrm{RR}$ \\
\hline $\begin{array}{l}\text { WiLLIAMS, } 2003 \\
\text { [58] }\end{array}$ & Crossover & $16(94)$ & $\mathrm{CHF}(16)$ & $61 \pm 8.8$ & Diamorphine & 1 or $2 \mathrm{mg}$ & Parenteral & Placebo & $\begin{array}{l}\text { Single } \\
\text { dose }\end{array}$ & Unclear & $P_{\mathrm{ETCO}_{2}}, \mathrm{RR}$ \\
\hline $\begin{array}{l}\text { Woodcock, } \\
1982 \text { [37] }\end{array}$ & Crossover & 16 & COPD (16) & No data & Dihydrocodeine & 90 or $180 \mathrm{mg} \cdot \mathrm{day}^{-1}$ & Oral & Placebo & 2 weeks & Outpatient & $\mathrm{PaCO}_{2}, \mathrm{PaO}_{2}$ \\
\hline $\begin{array}{l}\text { ALLCROFT, } 2013 \\
\text { [59] }\end{array}$ & Nonrandomised & $13(62)$ & COPD (13) & $\begin{array}{c}\text { Median } 78 \\
\text { (range 68-89) }\end{array}$ & Morphine & $10 \mathrm{mg} \cdot \mathrm{day}^{-1}$ & Oral & & 4 days & $\begin{array}{l}\text { Inpatient/ } \\
\text { outpatient }\end{array}$ & $\begin{array}{c}P_{\mathrm{ETCO}_{2}} \\
\mathrm{SaO}_{2}, \mathrm{RR} \\
\mathrm{RD}\end{array}$ \\
\hline $\begin{array}{l}\text { Boyd, } 1997 \\
{[60]}\end{array}$ & Nonrandomised & $15(47)$ & Cancer (15) & $\begin{array}{l}73 \text { (range } \\
62-85 \text { ) }\end{array}$ & Morphine & $\begin{array}{l}20 \mathrm{mg} \cdot \text { day }^{-1} \text { or } 130 \% \\
\text { of pretreatment dose }\end{array}$ & Oral & & $7-10$ days & $\begin{array}{l}\text { Inpatient/ } \\
\text { outpatient }\end{array}$ & $\mathrm{RR}$ \\
\hline $\begin{array}{l}\text { BRUERA, } 1990 \\
\text { [61] }\end{array}$ & Nonrandomised & $20(55)$ & Cancer (20) & $64 \pm 17$ & Morphine & $\begin{array}{l}5 \mathrm{mg} \text { or } 2.5 \text { times } \\
\text { pretreatment dose }\end{array}$ & Parenteral & & $\begin{array}{l}\text { Single } \\
\text { dose }\end{array}$ & Inpatient & $\begin{array}{l}P \mathrm{ETCO}_{2} \\
\mathrm{SaO}_{2}, \mathrm{RR}\end{array}$ \\
\hline $\begin{array}{l}\text { BRUERA, } 1993 \\
\text { (part 2) } \\
\text { [26] }\end{array}$ & Nonrandomised & 45 & Cancer (45) & No data & Morphine $^{+}$ & $\begin{array}{c}\text { Same dose as for } \\
\text { pain treatment }\end{array}$ & Parenteral & & $\begin{array}{l}\text { Total of } \\
312 \text { doses }\end{array}$ & Unclear & $\mathrm{RD}$ \\
\hline $\begin{array}{l}\text { Clemens, } 2007 \\
\text { [62] }\end{array}$ & Nonrandomised & $25(44)$ & Cancer (25) & $65.5 \pm 15.1$ & $\begin{array}{c}\text { Morphine } \\
\text { Hydromorphone }\end{array}$ & $\begin{array}{l}8.2(7.5) \mathrm{mg} \text { MED } \\
19.5(1.8) \mathrm{mg} \text { MED }\end{array}$ & No data & & $\begin{array}{l}\text { Single } \\
\text { dose }\end{array}$ & Inpatient & $\begin{array}{l}\mathrm{SaO}_{2}, \mathrm{RR} \\
\mathrm{RD}\end{array}$ \\
\hline $\begin{array}{l}\text { Clemens, } 2008 \\
\text { (1) [63] }\end{array}$ & Nonrandomised & $6(67)$ & $\operatorname{ALS}(6)$ & $57.0 \pm 6.9$ & Morphine ${ }^{\#}$ & $6.3(7.0) \mathrm{mg}$ & Oral & & $\begin{array}{l}\text { Single } \\
\text { dose }\end{array}$ & Inpatient & $\begin{array}{l}\mathrm{PaCO}_{2}, \mathrm{SaO}_{2}, \\
\mathrm{RR}, \mathrm{RD}\end{array}$ \\
\hline $\begin{array}{l}\text { Clemens, } 2008 \\
\text { (2) [64] }\end{array}$ & Nonrandomised & $14(57)$ & Cancer (14) & $\begin{array}{c}\text { Median } 67 \\
\text { (range } 40-84 \text { ) }\end{array}$ & Hydromorphone ${ }^{\#}$ & $2.5(1.8) \mathrm{mg}$ & Oral & & $\begin{array}{l}\text { Single } \\
\text { dose }\end{array}$ & Inpatient & $\begin{array}{l}\mathrm{PaCO}_{2}, \mathrm{SaO}_{2}, \\
\mathrm{RR}, \mathrm{RD}\end{array}$ \\
\hline $\begin{array}{l}\text { Clemens, } 2008 \\
\text { (3) [65] }\end{array}$ & Nonrandomised & $27(48)$ & $\begin{array}{l}\text { Cancer (25) } \\
\text { ALS (2) }\end{array}$ & Range 40-90 & $\begin{array}{c}\text { Morphine }{ }^{\#, \# \#} \\
\text { Hydromorphone }\end{array}$ & $\begin{array}{c}2.5-20.0 \mathrm{mg} \\
0.5-6.0 \mathrm{mg}\end{array}$ & Oral & & $\begin{array}{l}\text { Single } \\
\text { dose }\end{array}$ & Inpatient & $\begin{array}{l}\mathrm{PaCO}_{2}, \mathrm{SaO}_{2}, \\
\mathrm{RR}, \mathrm{RD}\end{array}$ \\
\hline $\begin{array}{l}\text { Clemens, } 2009 \\
\text { [66] }\end{array}$ & Nonrandomised & $46(54)$ & Cancer (46) & Range 40-90 & $\begin{array}{c}\text { Morphine } \#, \# \# \\
\text { Hydromorphone }\end{array}$ & $\begin{array}{l}2.5-20 \mathrm{mg} \\
1-6 \mathrm{mg}\end{array}$ & Oral & & $\begin{array}{l}\text { Single } \\
\text { dose }\end{array}$ & Inpatient & $\begin{array}{l}\mathrm{PaCO}_{2}, \mathrm{SaO}_{2}, \\
\mathrm{RR}, \mathrm{RD}\end{array}$ \\
\hline $\begin{array}{l}\text { CLemens, } 2011 \\
\text { [67] }\end{array}$ & Nonrandomised & $26(54)$ & Cancer (26) & $66.0 \pm 13.6$ & $\begin{array}{c}\text { Morphine } \\
\text { Hydromorphone }\end{array}$ & $\begin{array}{c}8.4(7.2) \mathrm{mg} \\
4(4.7) \mathrm{mg}\end{array}$ & Oral & & $\begin{array}{l}\text { Single } \\
\text { dose }\end{array}$ & Inpatient & $\begin{array}{l}\mathrm{PaCO}_{2}, \mathrm{SaO}_{2}, \\
\mathrm{RR}, \mathrm{RD}\end{array}$ \\
\hline $\begin{array}{l}\text { CoHEN, } 1991 \\
\text { [68] }\end{array}$ & Nonrandomised & 8 & Cancer (8) & $\begin{array}{l}61.9 \text { (range } \\
50-79 \text { ) }\end{array}$ & Morphine & $120 \mathrm{mg} \cdot \mathrm{day}^{-1}$ & Parenteral & & $60 \mathrm{~h}$ & Unclear & $\begin{array}{c}\mathrm{PaCO}_{2} \\
\mathrm{PaO}_{2}, \mathrm{RR}\end{array}$ \\
\hline $\begin{array}{l}\text { CoYne, } 2002 \\
\text { [69] }\end{array}$ & Nonrandomised & $35(43)$ & $\begin{array}{c}\text { Cancer (33) } \\
\text { Pulmonary } \\
\text { embolism (1) } \\
\text { AIDS (1) }\end{array}$ & 56 & Fentanyl & $25 \mu \mathrm{g}$ & Nebulised & & $\begin{array}{l}\text { Single } \\
\text { dose }\end{array}$ & Inpatient & $\mathrm{SaO}_{2}, \mathrm{RR}$ \\
\hline
\end{tabular}




\begin{tabular}{|c|c|c|c|c|c|c|c|c|c|c|c|}
\hline $\begin{array}{l}\text { First author, } \\
\text { year } \\
\text { [reference] }\end{array}$ & Design & $\begin{array}{c}\text { Subjects } \\
\text { n (\% } \\
\text { male) }\end{array}$ & $\begin{array}{l}\text { Population } \\
\text { (n) }\end{array}$ & Age years & Opioid & Dose & Administration & Comparison & Duration & $\begin{array}{l}\text { Patient } \\
\text { setting }\end{array}$ & $\begin{array}{l}\text { Included } \\
\text { outcomes }\end{array}$ \\
\hline $\begin{array}{l}\text { CuRRow, } 2011 \\
\text { [70] }\end{array}$ & Nonrandomised & $83(64)$ & $\begin{array}{l}\text { COPD (45) } \\
\text { Cancer (24) } \\
\text { ILD (10) } \\
\text { Other (4) }\end{array}$ & $74.6 \pm 9.1$ & Morphine & $\begin{array}{c}\text { Target: } 10-30 \mathrm{mg} \\
\text { Phase II: } 16.5 \text { (8) mg } \\
\text { Phase IV: } 14.0 \text { (6.3) } \\
\text { mg }\end{array}$ & Oral & & $\begin{array}{l}\text { Target } \\
3 \text { months } \\
\text { (mean } \\
142 \text { days) }\end{array}$ & Outpatient & $\mathrm{RD}$ \\
\hline $\begin{array}{l}\text { GaunA, } 2008 \\
\text { [71] }\end{array}$ & Nonrandomised & $4(50)$ & $\begin{array}{l}\text { COPD and } \\
\text { PF (2) } \\
\text { Cancer (2) }\end{array}$ & Range 52-85 & Fentanyl $^{+}$ & $200-400 \mu g$ & Oral & & $\begin{array}{l}\text { Single } \\
\text { dose }\end{array}$ & Inpatient & $\mathrm{SaO}_{2}, \mathrm{RR}$ \\
\hline $\begin{array}{l}\text { Munck, } 1990 \\
\text { (part 1) } \\
{[50]}\end{array}$ & Nonrandomised & 21 & COPD (21) & $\begin{array}{c}\text { Median } 67 \\
\text { (range } 50-78 \text { ) }\end{array}$ & Codeine & 60 and $120 \mathrm{mg}$ & Oral & & $\begin{array}{l}\text { Single } \\
\text { dose }\end{array}$ & Outpatient & $\begin{array}{c}\mathrm{PaCO}_{2,} \\
\mathrm{PaO}_{2}, \mathrm{SaO}_{2,} \\
\mathrm{RR}, \mathrm{RD}\end{array}$ \\
\hline $\begin{array}{l}\text { Otulana, } 2004 \\
\text { (phase 4) } \\
\text { [53] }\end{array}$ & Nonrandomised & 6 & Asthma (6) & No data & Morphine & $17.6 \mathrm{mg}$ & Nebulised & & $\begin{array}{l}\text { Single } \\
\text { dose }\end{array}$ & Unclear & $\mathrm{RR}$ \\
\hline $\begin{array}{l}\text { TANAKA, } 1999 \\
\text { [72] }\end{array}$ & Nonrandomised & $15(53)$ & Cancer (15) & $\begin{array}{c}\text { Median } 61 \\
\text { (range 42-76) }\end{array}$ & Morphine & $20 \mathrm{mg}$ & Nebulised & & $\begin{array}{l}\text { Single } \\
\text { dose }\end{array}$ & Inpatient & $\begin{array}{l}\mathrm{SaO}_{2}, \mathrm{RR} \\
\mathrm{RD}\end{array}$ \\
\hline \multicolumn{12}{|c|}{ 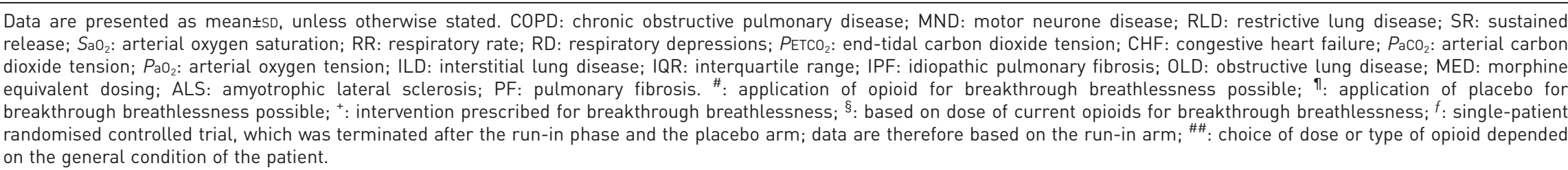 } \\
\hline
\end{tabular}


risk of bias of the NRTs. High risk of selection bias, performance bias and detection bias were estimated, as no control condition was included in these studies. In the other categories, the risk of bias was assessed as low in most studies. The POSs were graded with three to six out of eight stars due to comparability and representativeness of cohorts (online supplementary table S7).

\section{Effect on outcomes of respiratory adverse effects}

The effects of opioid treatment on outcomes of respiratory adverse effects are shown in online supplementary tables S2, S3, S8 and S9. A summary of the effects of the RCTs included in the meta-analyses is presented in table 2. Since none of the intraclass correlation coefficients of comparisons within RCTs were significantly different from zero, and therefore the effect of clustering on the outcomes was negligible for RCTs that contributed more than one contrast for a single outcome measure, the results are analysed using regular meta-analyses instead of three-level meta-analyses. Most of the included RCTs were crossover trials and we included both parallel and crossover trials in the meta-analyses together. Results of 12 RCTs could not be included in the meta-analyses because they compared opioid treatment to something other than placebo (treatment with another substance $[50,52,54]$, another dose or route of administration [43, 45, 48,53] or usual care [56] (online supplementary table S8)). Results of seven RCTs could not be included in the meta-analyses because they reported their outcomes as median scores [51], did not report the outcomes per treatment arm $[46,49]$ or reported the outcome in qualitative wording only $[30,44,55,57]$ (online supplementary table S8).

\section{Effect on $\mathrm{PaCO}_{2}$}

The effect of opioid treatment on $\mathrm{PaCO}_{2}$ was assessed in nine RCTs [29, 33, 37, 50, 51, 54-56], five of which could be included in the meta-analysis $[29,33,37,51]$. The meta-analysis showed that treatment

TABLE 2 Summary of findings: opioids compared to placebo for patients with chronic breathlessness due to advanced disease in inpatient and outpatient settings

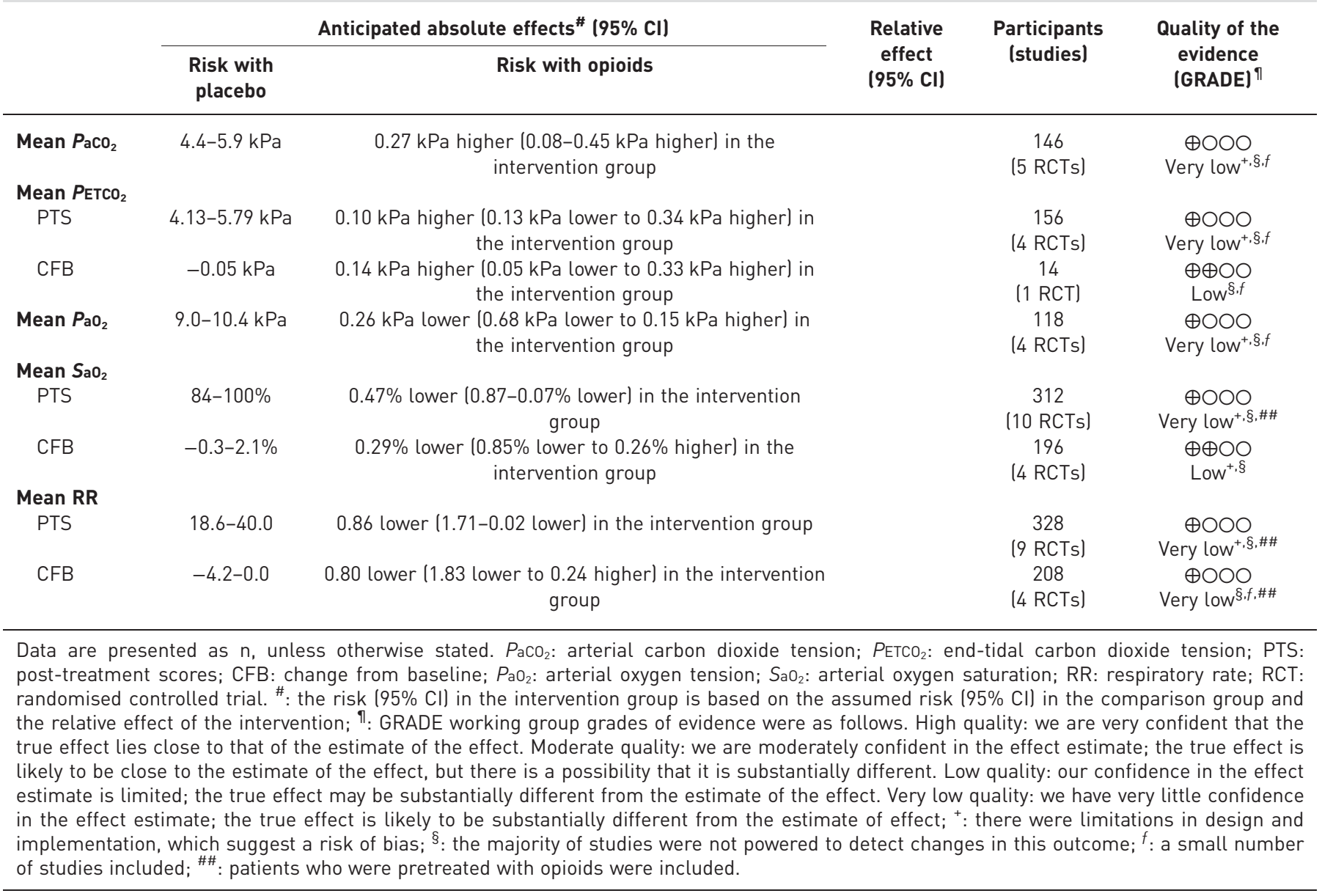


with opioids increased $\mathrm{PaCO}_{2}$ (MD 0.27, 95\% CI 0.08-0.45; $\mathrm{I}^{2} 0 \%$; figure 2a). The meta-regression revealed no influence from the context of assessment ( $\mathrm{p}=0.437$; however, there was only one RCT during exercise) or the number of doses $(\mathrm{p}=0.507)$ on the $\mathrm{PaCO}_{2}$. Route of administration was not taken into account, since all RCTs administered the opioid systemically. One RCT examined the effect of opioids on $\mathrm{PaCO}_{2}$ during exercise [33]. The difference between the intervention and control groups after administration of morphine was statistically significant at maximal exercise (5.8 and $5.1 \mathrm{kPa}$, respectively; $\mathrm{p}<0.001$ ).

The effect on $\mathrm{PaCO}_{2}$ was assessed in seven NRTs [50, 63-68]. One NRT found a significant increase in $P_{\mathrm{aCO}_{2}}$ [68]. Finally, the effect on $\mathrm{PaCO}_{2}$ was assessed in one ROS [73] and one case report describing two cases [74]. In both studies the opioids were nebulised. The opioids were prescribed as a single dose or for up to 15 days. In all studies, $\mathrm{PaCO}_{2}$ was measured at rest. None of these studies showed a significant effect of opioid treatment on $\mathrm{PaCO}_{2}$.

\section{Effect on $\mathrm{PETCO}_{2}$}

The effect of opioid treatment on $\mathrm{PETCO}_{2}$ was assessed in seven RCTs [27, 29, 30, 32, 34, 44, 58], five of which could be included in the meta-analysis [27, 29, 32, 34, 58]. The meta-analysis showed a nonsignificant increase of the $\mathrm{PETCO}_{2}$ (MD 0.13, 95\% CI $-0.02-0.27 ; \mathrm{I}^{2} 0 \%$; figure $2 \mathrm{~b}$ ). The RCT by LigHT et al. [34] had a low variance compared to the other studies, and consequently a high weight in the analysis. Therefore, as a sensitivity analysis, the meta-analysis was repeated, but with weighing based on the sample size. The effect on $\mathrm{PETCO}_{2}$ was still not significant (MD 0.13, 95\% CI $-0.11-0.37 ; \mathrm{I}^{2} 0 \%$ ). The meta-regression revealed no influence from the context of assessment $(p=0.375)$, the number of doses $(\mathrm{p}=0.679)$ or the route of administration $(\mathrm{p}=0.473)$ on the $\mathrm{PETCO}_{2}$.

The effect on $\mathrm{PETCO}_{2}$ was assessed in two NRTs $[59,61]$. These studies reported no significant change in $\mathrm{PETCO}_{2}[59,61]$.

a)

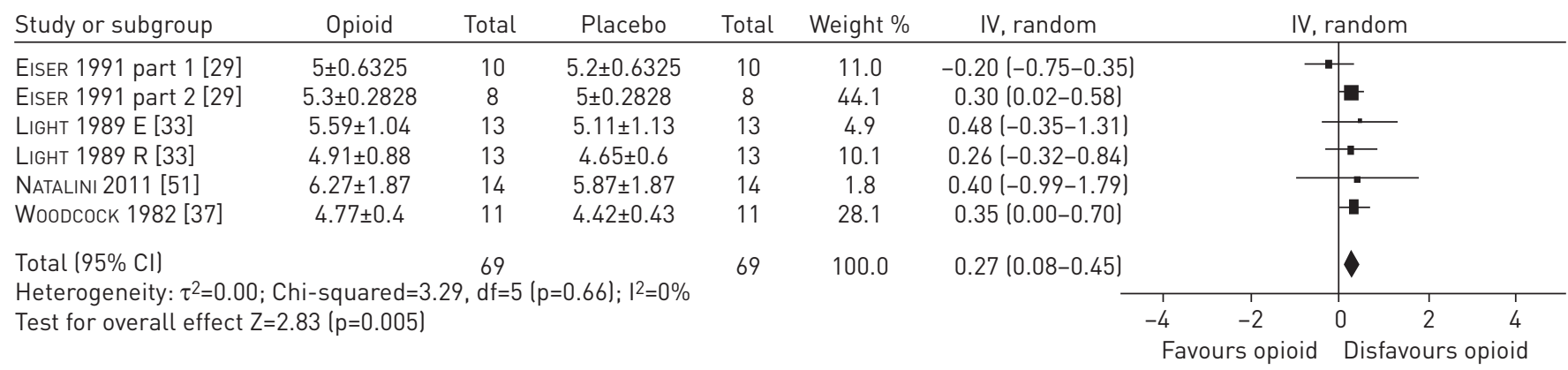

b)

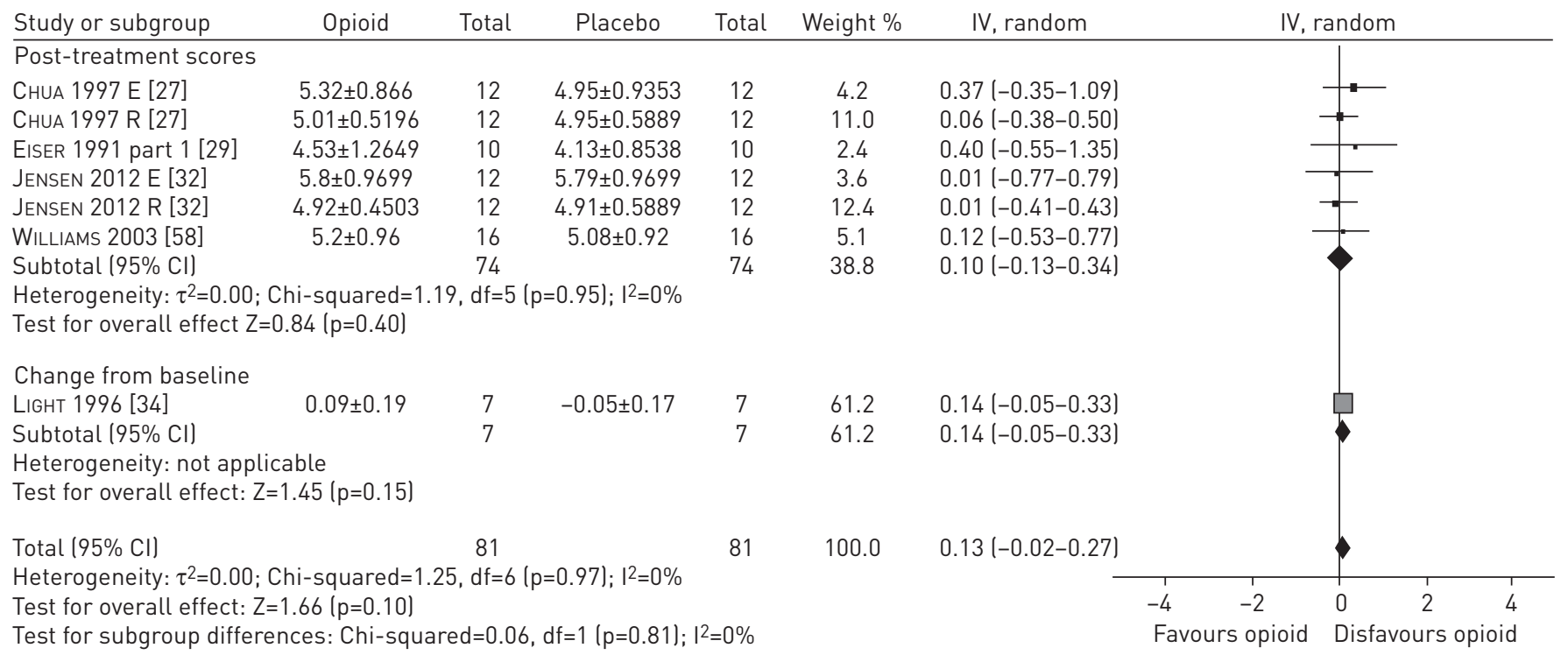

FIGURE 2 Effect of opioid treatment in patients with advanced disease on al arterial carbon dioxide tension; b) end-tidal carbon dioxide tension. Data are presented as mean \pm SD or mean difference $(95 \%$ CI), unless otherwise stated. E: measured on exertion; R: measured at rest. 
Effect on $\mathrm{PaO}_{2}$

The effect of opioid treatment on $\mathrm{PaO}_{2}$ was assessed in nine RCTs [29, 33, 37, 50, 51, 54-56], four of which could be included in the meta-analysis $[29,33,37]$. The meta-analysis showed a nonsignificant decrease of the $\mathrm{PaO}_{2}\left(\mathrm{MD}-0.26,95 \% \mathrm{CI}-0.68-0.15 ; \mathrm{I}^{2} 0 \%\right.$; figure 3$)$. The meta-regression revealed no influence from the context of assessment $(p=0.420$; however, only one RCT was conducted during exercise) or the number of doses $(\mathrm{p}=0.815)$ on the $\mathrm{PaO}_{2}$. Route of administration was not taken into account, since all RCTs administered the opioid systemically. One RCT examined the effect of opioids on $\mathrm{PaO}_{2}$ during exercise [33]. The difference between the intervention and control groups after administration of morphine was statistically significant at maximal exercise $(8.8$ and $9.6 \mathrm{kPa}$, respectively; $\mathrm{p}<0.05)$.

The effect on $\mathrm{PaO}_{2}$ was assessed in two NRTs $[50,68]$. One NRT found a significant decrease in $\mathrm{PaO}_{2}[68]$. Finally, the effect on $\mathrm{PaO}_{2}$ was assessed in one case report describing two cases [74]. In this study the opioids were nebulised for up to 15 days. $\mathrm{PaO}_{2}$ was measured at rest. This study showed no significant effect of opioid treatment on $\mathrm{PaO}_{2}$.

\section{Effect on $\mathrm{SaO}_{2}$}

The effect of opioid treatment on $\mathrm{SaO}_{2}$ was assessed in 24 RCTs [25-33, 35, 38-42, 44-46, 48-50, 52, 53, 57], 14 of which could be included in the meta-analysis [26-33, 35, 38-42]. The meta-analysis showed that $\mathrm{SaO}_{2}$ decreased after opioid use (MD $-0.41,95 \% \mathrm{CI}-0.73--0.08 ; \mathrm{I}^{2} 0 \%$; figure 4$)$. The study by ChUA et al. [27] was the only RCT showing a significant difference in $\mathrm{SaO}_{2}$ between the intervention and control groups at rest $(99.3 \%$ and $100 \%$, respectively; $\mathrm{p}=0.03)$. This RCT reported a variance of zero in the control group in rest, and consequently had a high weight in the analysis. Therefore, as a sensitivity analysis the meta-analysis was repeated, but with weighing based on the sample size. The effect on $\mathrm{SaO}_{2}$ was no longer significant ( $\mathrm{MD}-0.31,95 \% \mathrm{CI}-1.06-0.45 ; \mathrm{I}^{2} 0 \%$ ). The meta-regression revealed no influence from the context of assessment $(\mathrm{p}=0.730)$, the number of doses $(\mathrm{p}=0.165)$ or the route of administration $(\mathrm{p}=0.538)$ on the $\mathrm{SaO}_{2}$.

Furthermore, the effect of opioids on $\mathrm{SaO}_{2}$ was assessed in 12 NRTs [50, 59, 61-67, 69, 71, 72]. One NRT showed a significant decrease in $\mathrm{SaO}_{2}$ from $93 \%$ to $92 \%$ [50] after a single dose of $120 \mathrm{mg}$ codeine. However, this decrease was temporary and not clinically relevant. Finally, the effect of opioids on $\mathrm{SaO}_{2}$ was assessed in two POSs [75, 76], two ROSs $[73,77]$ and three case reports describing seven cases $[74,78$, 79]. In these studies, the opioids were administered systemically $(\mathrm{n}=3)$, nebulised $(\mathrm{n}=2)$ or via an unknown route $(n=1)$. The opioids were prescribed as single dose or as repeated doses up to 3 months. In all studies, $\mathrm{SaO}_{2}$ was measured at rest. None of these studies showed a significant effect of opioid treatment on $\mathrm{SaO}_{2}$. In two RCTs [25, 44] and one NRT [59] $\mathrm{SaO}_{2}$ was measured, but no outcome data were reported.

\section{Effect on respiratory rate}

The effect of opioid treatment on respiratory rate was assessed in 23 RCTs [25-28, 30, 32, 33, 35, 36, 38$41,43,45-47,49-51,53,57,58], 13$ of which could be included in the meta-analysis $[25,26,30,32,33$, $35,36,38-41,47,58]$. The meta-analysis showed that treatment with opioids significantly decreased the respiratory rate $\left(\mathrm{MD}-1.10,95 \% \mathrm{CI}-1.49--0.71 ; \mathrm{I}^{2} 0 \%\right.$; figure 5$)$. The study by SHOHRATI et al. [36] was the only RCT showing a significant difference in change in respiratory rate between the intervention and control groups $(-1.5$ and -0.1 , respectively; $\mathrm{p}<0.001)$. This RCT had a low variance compared to the other studies, and consequently a high weight in the analysis. Therefore, as a sensitivity analyis, the meta-analysis was repeated, but with weighing based on the sample size. The effect on respiratory rate was no longer significant ( $\mathrm{MD}-0.58,95 \% \mathrm{CI}-1.72-0.56 ; \mathrm{I}^{2} 0 \%$ ). The heterogeneity among the RCTs describing post-treatment scores was $0 \%$. The meta-regression revealed no influence from the context of

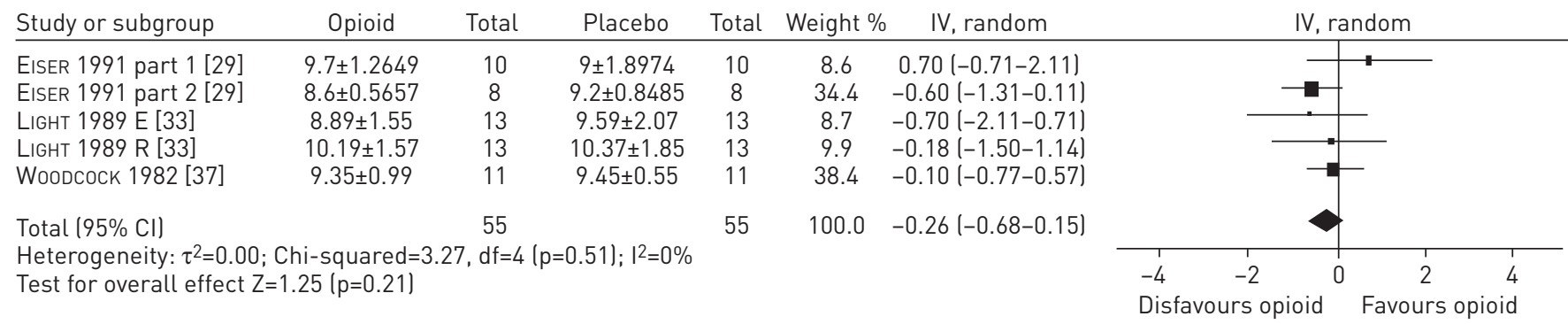




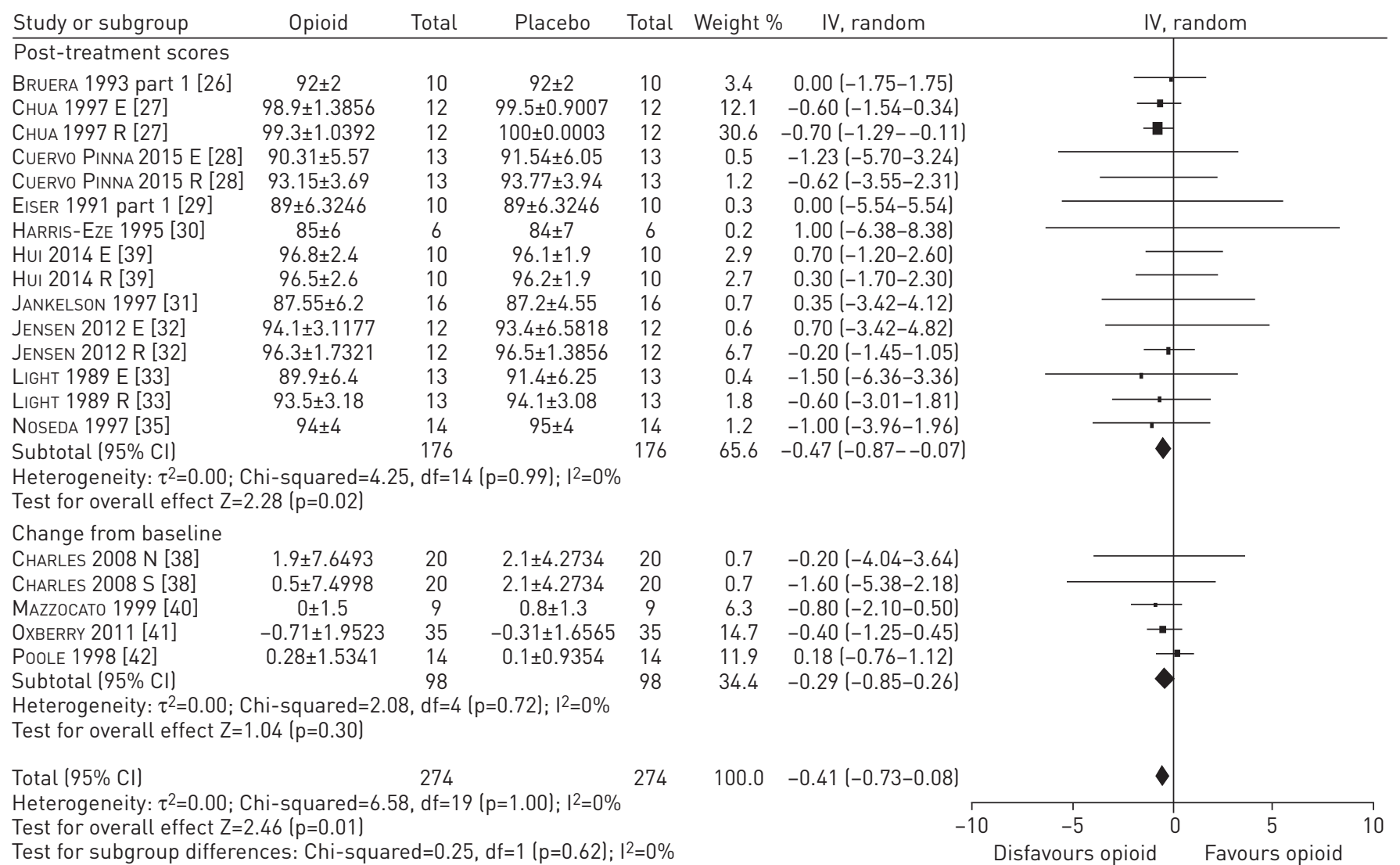

FIGURE 4 Effect of opioid treatment in patients with advanced disease on arterial oxygen saturation. E: measured on exertion; R: measured at rest.

assessment $(\mathrm{p}=0.496)$, the number of doses $(\mathrm{p}=0.904)$ or the route of administration $(\mathrm{p}=0.139)$ on the respiratory rate.

The effect on respiratory rate was also assessed in 15 NRTs [50, 53, 59-69, 71, 72]. These studies also showed that opioids caused no significant change in respiratory rate. Finally, the effect on respiratory rate was assessed in three POSs $[75,76,80]$, two ROSs $[73,77]$ and four case reports describing 10 cases [74, $78,79,81]$. In these studies, the opioids were administered systemically $(\mathrm{n}=4)$, nebulised $(\mathrm{n}=4)$ or via an unknown route $(n=1)$. The opioids were prescribed as single dose or as repeated doses up to 3 months. In all studies, $\mathrm{SaO}_{2}$ was measured at rest. These studies also showed that opioids caused no significant change in respiratory rate. In two RCTs $[28,50]$ and one NRT [50], respiratory rate was measured, but no outcome data were reported.

\section{Occurrence of respiratory depression}

The occurrence of respiratory depressions was reported in five RCTs $[25,32,40,45,56], 11$ NRTs $[26,50$, 59, 62-67, 70, 72], two POSs [14, 75], three ROSs [15, 82, 83] and four case reports describing 10 cases $[13,74,79,84]$. Out of these 25 studies, 11 defined respiratory depression [13, 14, 40, 63-67, 72, 75, 84]. Definitions were based on an increase in $\mathrm{PaCO}_{2}$ of $>0.5 \mathrm{kPa}$ or to $>6.0 \mathrm{kPa}$, a decrease in respiratory rate of $>10 \%$ or to $<10$ breaths $\mathrm{min}^{-1}$ and a decrease in $\mathrm{SaO}_{2}$ of $>5 \%$ or to $<90 \%$. Hu et al. [14] observed a case of respiratory depression (defined as decrease in respiratory rate to $<10$ breaths $\cdot \mathrm{min}^{-1}$ ) in one patient with terminal cancer both at the beginning of the POS and 2 days prior to death. KaWABATA and KaneisHi [15] reported three patients experiencing respiratory depressions (no definition given), which were not serious. It was not stated whether these patients were treated for pain or breathlessness. LANG and JEDEIKIN [13] described a case of respiratory depression (defined as respiratory rate of $4-5$ breaths $\cdot \mathrm{min}^{-1}$, very poor respiratory effort and minimal wheezing over both lung fields) after administration of $4 \mathrm{mg}$ nebulised morphine and $4 \mathrm{mg}$ dexamethasone for breakthrough breathlessness in a patient already using $10 \mathrm{mg}$ oral slow-release morphine three times per day and $10 \mathrm{mg}$ oral immediate-release morphine when required for cancer-related pain. 


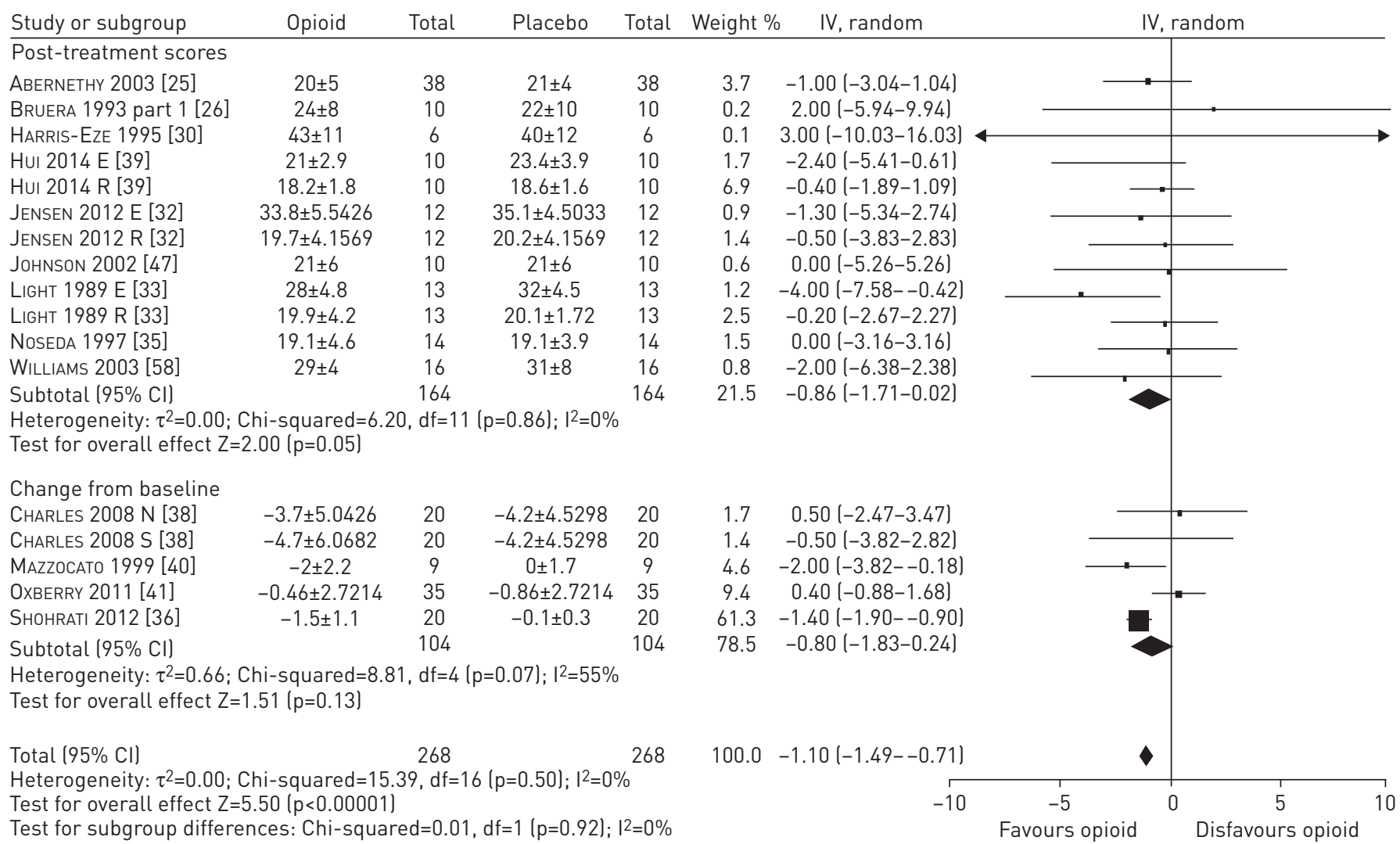

FIGURE 5 Effect of opioid treatment in patients with advanced disease on respiratory rate. E: measured on exertion; R: measured at rest; $\mathrm{N}$ : nebulised administration; S: systemic administration.

\section{Quality of the evidence}

The quality of the evidence was assessed as very low to moderate for the different outcomes (table 2). Only RCTs were included in this assessment. For all outcomes, the majority of the RCTs were small with insufficient power to assess respiratory adverse events and the quality was therefore downgraded. Furthermore, limitations in the design and implementation were observed. In several RCTs, patients who were pretreated with opioids were included, which had a negative effect on the quality of the evidence. Finally, only a small number of RCTs included assessment of $\mathrm{PaCO}_{2}$ and $\mathrm{PaO}_{2}$.

\section{Discussion \\ Main findings}

This systematic review on occurrence of respiratory adverse effects following opioid treatment for breathlessness shows a great heterogeneity of treatment regimens and patient populations. Given this heterogeneity, we found no evidence that clinically relevant respiratory adverse effects are to be expected in patients with breathlessness who are treated with opioids, while included studies confirmed previous reports of opioid-related benefit for breathlessness. This suggests that clinicians' fears of respiratory obtundation with the use of low-dose opioids seem to be unfounded.

The meta-analysis showed an increase in $\mathrm{PaCO}_{2}$ of $0.27(0.09-0.46) \mathrm{kPa}$. Although this increase is statistically significant, it is not considered to be clinically relevant [85]. Indeed, the pooled mean $\pm \mathrm{SD} \mathrm{PaCO}_{2}$ was $5.35 \pm 1.08 \mathrm{kPa}$, so the mean difference in $\mathrm{PaCO}_{2}$ was only $25 \%$ of the standard deviation. However, few RCTs reported on $\mathrm{PaCO}_{2}$ and the quality of this evidence is assessed as very low. One NRT reported a significant deterioration of blood gases, but the participants received $120 \mathrm{mg}$ parenteral morphine per day [68]. Given that $20-40 \%$ of oral morphine is bioavailable, this represents a much higher dose than the oral morphine doses required in the dose titration study (10-30 mg oral morphine per day) [70] or the oral morphine repeat dose trials $(20 \mathrm{mg}$ oral morphine per day) [25, 41, 47]. The meta-analyses showed a significant decrease in $\mathrm{SaO}_{2}$ of $0.41(0.73-0.08) \%$ and respiratory rate of 1.10 (1.49-0.71) breaths $\mathrm{min}^{-1}$. However, in both analyses one study had a high weighting due to a small variance. The statistical significance disappeared when the analyses were repeated weighted on sample size. In four cases, a 
diagnosis of respiratory depression was made during the study, but the definition was poorly stated. In three occasions the indication and dose were not clear [15]. In the fourth case, respiratory depression occurred in a patient with advanced metastatic cancer pretreated with opioids. The additive effect of both treatments, leading to a high dose of morphine, may have led to respiratory depression [13]. It is notable that no cases of respiratory depression were noted in the context of RCTs, with their close monitoring. Neither the meta-analyses of $\mathrm{EtCO}_{2}, \mathrm{PaO}_{2}, \mathrm{SaO}_{2}$ and respiratory rate nor the studies that were not included in the meta-analyses showed a significant deterioration of these outcomes. The meta-regression did not provide a significant effect for the context of assessment (at rest or on exertion), the number of doses (single dose or multiple doses) or the route of administration (nebulised or systemic), which is surprising especially for the route of administration. Previous reviews have reported a different effect of opioids on breathlessness when administered systemically or nebulised [5, 6]. The results of this meta-regression might be related to small effects within the included studies and the fact that only six studies included in the meta-analysis used nebulised opioid.

Six ongoing studies were identified. Three of those only examine a single or double dose of opioids and therefore will not illuminate the long-term effect. Of the three other studies, two have respiratory adverse effects as a secondary outcome and the sample size calculation will therefore probably not be based on this outcome. Only the MORDYC (Morphine for Dyspnea in COPD) study focuses primarily on respiratory adverse effects and the sample size calculation is based on the $\mathrm{PaCO}_{2}$ [85]. This study will add valuable information about the occurrence of respiratory adverse effects.

Our findings are consistent with other reviews on opioids for chronic breathlessness $[5,6,86]$ and episodic breathlessness [87]. These reviews included RCTs [5, 6, 86, 87], NRTs [6, 86] and case reports [87]. The authors of these reviews found no clinically relevant effect on blood gases or oxygen saturation, or respiratory depression after treatment with different types of opioids in patients with advanced disease. In hypoxic patients with cancer, an improvement of $\mathrm{SaO}_{2}$ was reported [6]. However, these reviews only included 39 studies, and meta-analyses could not be performed due to limited results on respiratory adverse effects. Furthermore, the focus of these reviews was on the effect of opioid treatment on breathlessness and search terms for respiratory adverse effects were not included.

\section{Limitations of the included studies}

First, the risk of bias of the included studies was often difficult to estimate. The outcomes of interest in the current review were secondary outcomes in the majority of the included studies and therefore the method of outcome assessment was often not described. The method of randomisation or allocation concealment was inadequately described in most studies. Since it was difficult to score the risk of bias and to set a cut-off point, we did not include a sensitivity analysis including only the studies with a low risk of bias. Second, there was great heterogeneity in the dosing regimens and comparators used. The prescribed doses ranged between the studies, with eight studies prescribing high doses of opioids. In 34 experimental studies, one observational study and seven cases, only a single dose of opioids was prescribed, so the long-term effect was not assessed. Seven RCTs did not include a placebo group, but used different doses, other medication or usual care as comparator. Third, the patient populations were heterogeneous. In some studies, patients had to be opioid-naïve, but not in others: patients could continue opioids for pain or the dose of the study medication was based on current analgesic treatment. Fourth, the included studies had a small sample size. The experimental studies included between one and 83 participants, with only six studies including a sample size of $\geqslant 30$ participants per treatment group. These studies included outcomes of respiratory adverse effects, but were underpowered to properly assess a change in these outcomes. The observational studies used larger sample sizes, but only a proportion of these patients received opioids for breathlessness. In some studies, the results accounted for the entire group, making it impossible to draw conclusions for the subgroup of our interest. Fifth, the definition of respiratory depression differed between studies. The most reliable assessment of respiratory depression is based on the $\mathrm{PaO}_{2}$ and $\mathrm{PaCO}_{2}$. Measurement of $\mathrm{SaO}_{2}$ is less reliable [88]. Some authors included respiratory rate as a measure of respiratory depression, because this is easier to estimate. Only 11 studies defined respiratory depression and eight used a decrease in $\mathrm{SaO}_{2}$ as part of the definition. Only four also included an increase of $\mathrm{PaCO}_{2}$. Finally, five studies mentioned the assessment of respiratory outcomes in their method section, but didn't include the results $(n=3)$ or only reported the baseline data $(n=2)$. Furthermore, 25 studies reported on the occurrence of respiratory depression, but only nine of them mentioned the assessment of respiratory depression in their methods section. Therefore, it is not known if a respiratory depression occurred in any of the remaining 42 studies.

\section{Strengths and limitations of the current review}

Our study has several strengths. We included several study types; although RCTs yield the most reliable evidence, observational studies and case reports are closer to daily clinical practice. Furthermore, we 
included studies that were published in five languages. Because of the large number of included studies, we were able to present the current knowledge of six different outcomes of respiratory adverse effects and were able to perform meta-analyses on five of these. This provides an overall estimate of the effect of opioid treatment on these outcomes.

Our review has several limitations. First, we only searched four databases. Due to publication bias, we might have failed to identify negative results. However, we also searched one trial register, sought expert opinions and hand-searched the reference lists of important reviews in the field of opioid treatment for chronic breathlessness. We identified a large number of studies, decreasing the chance that we missed important studies. Second, several RCTs could not be included in the meta-analyses because of reasons discussed earlier. Third, we combined results from studies with different contexts of assessment, different number of doses and different route of administration; however, this was done only after the meta-regression which did not yield evidence that these moderators had an effect on the outcome. The number of studies used for this analysis was in some cases very low, making the power to detect effects questionable. However, due to the robustness of the results (i.e. no single moderator was significant in any of the analyses), we combined all measures to be pooled. Fourth, the patient populations were too diverse to specify the results for different populations. We primarily expect that patients with COPD and chronic respiratory failure are more at risk of respiratory adverse effects than patients with cancer or heart failure, for example. Most of the studies included patients with a specified primary diagnosis $(n=54)$, of which 16 studies only included patients with COPD. However, from these populations it is not known which patients experienced chronic respiratory failure. Fifth, we used the Cochrane risk of bias tool to assess the risk of bias in RCTs and NRTs. This tool is designed for use in RCTs, but there was no appropriate alternative to use in NRTs. After assessment of the risk of bias was completed, the Risk of Bias in Nonrandomized Studies - of Interventions (ROBINS-I) tool was published [89]. This might have been a better tool to assess the risk of bias in NRTs and can be used in future studies. Finally, we included both crossover trials and parallel trials in the meta-analysis together and analysed the crossover trials as if they were parallel trials. This might result in a unit of analysis error, leading to an underweighting of the crossover trials. Since only two studies included in the meta-analyses of $\mathrm{SaO}_{2}$ and respiratory rate were parallel trials and the remaining studies were crossover trials, we assume this influence to be negligible.

\section{Implications for clinical practice and future research}

Patients are willing to consider opioid treatment for chronic breathlessness, despite the occurrence of adverse effects, and report improvement of quality of life and relief of breathlessness as their main reasons [12]. However, physicians remain reluctant to prescribe opioids for chronic breathlessness, because of fear of adverse clinical outcomes, among others [9-12]. A recent large observational study of older adults with COPD by VOzonis et al. [90] showed an association between new prescription of opioid and a small, but statistically significant increase in 30-day mortality and emergency visits. However, palliative care patients (and thus those who form the main group for whom opioids would be prescribed for breathlessness) were excluded and other differences between patients with and without opioid use might explain these findings. In contrast, a registry study of people with advanced COPD on long-term oxygen therapy, with 4 years' follow-up found no association with either hospital admission or survival in people taking $\leqslant 30 \mathrm{mg}$ of oral morphine per day [91].

This review has shown that the current evidence on respiratory adverse effects of opioid treatment in chronic breathlessness is inconsistent and heterogenic. Only one serious episode of respiratory depression is described, and that in the context of high-dose opioids. Based on the evidence included in this review, low-dose opioids can be considered as safe treatment for chronic breathlessness in the context of good clinical care and appropriate monitoring. However, the studies that have been conducted are mostly of low quality, short duration and not designed to assess the effect of low-dose opioids on respiratory adverse effects. A long-term, well-powered randomised controlled trial, such as the MORDYC study, is needed. Moreover, including a common respiratory outcome set in all trials of opioids for breathlessness, so that a more robust synthesis could be conducted, is recommended.

\section{Acknowledgements}

We would like to thank Lowie E.W.G. Vanfleteren (Centre of Expertise for Chronic Organ Failure and Maastricht University Medical Centre, Maastricht, the Netherlands) for contributing to the screening of and data extraction from the articles in the French and Spanish language.

\section{References}

1 Parshall MB, Schwartzstein RM, Adams L, et al. An official American Thoracic Society statement: update on the mechanisms, assessment, and management of dyspnea. Am J Respir Crit Care Med 2012; 185: 435-452.

2 Thomas S, Bausewein C, Higginson I, et al. Breathlessness in cancer patients - implications, management and challenges. Eur J Oncol Nurs 2011; 15: 459-469. 
3 Janssen DJ, Spruit MA, Wouters EF, et al. Daily symptom burden in end-stage chronic organ failure: a systematic review. Palliat Med 2008; 22: 938-948.

4 Johnson MJ, Yorke J, Hansen-Flaschen J, et al. Towards an expert consensus to delineate a clinical syndrome of chronic breathlessness. Eur Respir J 2017; 49: 1602277.

5 Ekström M, Bajwah S, Bland JM, et al. One evidence base; three stories: do opioids relieve chronic breathlessness? Thorax 2017; In press. https://doi.org/10.1136/thoraxjnl-2016-209868.

6 Vargas-Bermúdez A, Cardenal F, Porta-Sales J. Opioids for the management of dyspnea in cancer patients: evidence of the last 15 years - a systematic review. J Pain Palliat Care Pharmacother 2015; 29: 341-352.

7 Barnes H, McDonald J, Smallwood N, et al. Opioids for the palliation of refractory breathlessness in adults with advanced disease and terminal illness. Cochrane Database Syst Rev 2016; 3: CD011008.

8 Ekström M, Nilsson F, Abernethy AA, et al. Effects of opioids on breathlessness and exercise capacity in chronic obstructive pulmonary disease. A systematic review. Ann Am Thorac Soc 2015; 12: 1079-1092.

9 Hadjiphilippou S, Odogwu SE, Dand P. Doctors' attitudes towards prescribing opioids for refractory dyspnoea: a single-centred study. BMJ Support Palliat Care 2014; 4: 190-192.

10 Janssen DJ, de Hosson S, bij de Vaate E, et al. Attitudes toward opioids for refractory dyspnea in COPD among Dutch chest physicians. Chron Respir Dis 2015; 12: 85-92.

11 Young J, Donahue M, Farquhar M, et al. Using opioids to treat dyspnea in advanced COPD: attitudes and experiences of family physicians and respiratory therapists. Can Fam Physician 2012; 58: e401-e407.

12 Rocker G, Young J, Donahue M, et al. Perspectives of patients, family caregivers and physicians about the use of opioids for refractory dyspnea in advanced chronic obstructive pulmonary disease. CMAJ 2012; 184: E497-E504.

13 Lang E, Jedeikin R. Acute respiratory depression as a complication of nebulised morphine. Can J Anaesth 1998; 45: 60-62.

$14 \mathrm{Hu}$ WY, Chiu TY, Cheng SY, et al. Morphine for dyspnea control in terminal cancer patients: is it appropriate in Taiwan? J Pain Symptom Manage 2004; 28: 356-363.

15 Kawabata M, Kaneishi K. Continuous subcutaneous infusion of compound oxycodone for the relief of dyspnea in patients with terminally ill cancer: a retrospective study. Am J Hosp Palliat Care 2013; 30: 305-311.

16 Politis J, Le B, Smallwood N. Respiratory depression secondary to morphine use in a patient with COPD and refractory breathlessness. Eur Respir J 2017; 49: 1601858.

17 Lanken PN, Terry PB, Delisser HM, et al. An official American Thoracic Society clinical policy statement: palliative care for patients with respiratory diseases and critical illnesses. Am J Respir Crit Care Med 2008; 177: 912-927.

18 Integraal Kankercentrum Nederland. Dyspneu in de Palliatieve Fase 3.0 [Dyspnoea in the Palliative Phase 3.0]. www.oncoline.nl/dyspneu in de palliatieve fase.pdf Date last updated December 22, 2015.

19 Long Alliantie Nederland. Richtlijn Palliatieve zorg voor mensen met COPD [Guideline Palliative Care for People with COPD]. http:/nvalt.nl/uploads/gP/TM/gPTMm_YjhQB20AIn66wTng/LAN-Richtlijn-Palliatieve=Zorg-COPD. pdf Date last accessed: December 3, 2014. Date last updated: 2011.

20 Global Initiative for Chronic Obstructive Lung Disease (GOLD). Global Strategy for the Diagnosis, Management, and Prevention of Chronic Obstructive Pulmonary Disease, 2017 Report. http://goldcopd.org/gold-2017-globalstrategy-diagnosis-management-prevention-copd Date last accessed: March 2, 2017.

21 Higgins JPT, Green S. Cochrane Handbook for Systematic Reviews of Interventions version 5.1.0. The Cochrane Collaboration 2011. http://handbook-5-1.cochrane.org Date last accessed April 11, 2016.

22 Moher D, Liberati A, Tetzlaff J, et al. Preferred reporting items for systematic reviews and meta-analyses: the PRISMA statement. Int J Surg 2010; 8: 336-341.

23 Wells GA, Shea B, O'Connell D, et al. The Newcastle-Ottawa Scale (NOS) for Assessing the Quality of Nonrandomised Studies in Meta-analyses. University of Ottawa. www.ohri.ca/programs/clinical_epidemiology/ oxford.asp Date last accessed: May 12, 2016. Date last updated: January 2000.

24 Van den Noortgate W, López-López JA, Marín-Martínez F, et al. Three-level meta-analysis of dependent effect sizes. Behav Res Methods 2013; 45: 576-594.

25 Abernethy AP, Currow DC, Frith P, et al. Randomised, double blind, placebo controlled crossover trial of sustained release morphine for the management of refractory dyspnoea. BMJ 2003; 327: 523-528.

26 Bruera E, MacEachern T, Ripamonti C, et al. Subcutaneous morphine for dyspnea in cancer patients. Ann Intern Med 1993; 119: 906-907.

27 Chua TP, Harrington D, Ponikowski P, et al. Effects of dihydrocodeine on chemosensitivity and exercise tolerance in patients with chronic heart failure. J Am Coll Cardiol 1997; 29: 147-152.

28 Cuervo Pinna MÁ, Bruera E, Moralo MJR, et al. A randomized crossover clinical trial to evaluate the efficacy of oral transmucosal fentanyl citrate in the treatment of dyspnea on exertion in patients with advanced cancer. Am J Hosp Palliat Care 2015; 32: 298-304.

29 Eiser N, Denman WT, West C, et al. Oral diamorphine: lack of effect on dyspnoea and exercise tolerance in the "pink puffer" syndrome. Eur Respir J 1991; 4: 926-931.

30 Harris-Eze AO, Sridhar G, Clemens RE, et al. Low-dose nebulized morphine does not improve exercise in interstitial lung disease. Am J Respir Crit Care Med 1995; 152: 1940-1945.

31 Jankelson D, Hosseini K, Mather LE, et al. Lack of effect of high doses of inhaled morphine on exercise endurance in chronic obstructive pulmonary disease. Eur Respir J 1997; 10: 2270-2274.

32 Jensen D, Alsuhail A, Viola R, et al. Inhaled fentanyl citrate improves exercise endurance during high-intensity constant work rate cycle exercise in chronic obstructive pulmonary disease. J Pain Symptom Manage 2012; 43: 706-719.

33 Light RW, Muro JR, Sato RI, et al. Effects of oral morphine on breathlessness and exercise tolerance in patients with chronic obstructive pulmonary disease. Am Rev Respir Dis 1989; 139: 126-133.

34 Light RW, Stansbury DW, Webster JS. Effect of $30 \mathrm{mg}$ of morphine alone or with promethazine or prochlorperazine on the exercise capacity of patients with COPD. Chest 1996; 109: 975-981.

35 Noseda A, Carpiaux JP, Markstein C, et al. Disabling dyspnoea in patients with advanced disease: lack of effect of nebulized morphine. Eur Respir J 1997; 10: 1079-1083.

36 Shohrati M, Ghanei M, Harandi AA, et al. Effect of nebulized morphine on dyspnea of mustard gas-exposed patients: a double-blind randomized clinical trial study. Pulm Med 2012; 2012: 610921. 
37 Woodcock AA, Johnson MA, Geddes DM. Breathlessness, alcohol, and opiates. N Engl J Med 1982; 306: 1363-1364.

38 Charles MA, Reymond L, Israel F. Relief of incident dyspnea in palliative cancer patients: a pilot, randomized, controlled trial comparing nebulized hydromorphone, systemic hydromorphone, and nebulized saline. J Pain Symptom Manage 2008; 36: 29-38.

39 Hui D, Xu A, Frisbee-Hume S, et al. Effects of prophylactic subcutaneous fentanyl on exercise-induced breakthrough dyspnea in cancer patients: a preliminary double-blind, randomized, controlled trial. J Pain Symptom Manage 2014; 47: 209-217.

40 Mazzocato $\mathrm{C}$, Buclin $\mathrm{T}$, Rapin $\mathrm{CH}$. The effects of morphine on dyspnea and ventilatory function in elderly patients with advanced cancer: a randomized double-blind controlled trial. Ann Oncol 1999; 10: 1511-1514.

41 Oxberry SG, Torgerson DJ, Bland JM, et al. Short-term opioids for breathlessness in stable chronic heart failure: a randomized controlled trial. Eur J Heart Fail 2011; 13: 1006-1012.

42 Poole PJ, Veale AG, Black PN. The effect of sustained-release morphine on breathlessness and quality of life in severe chronic obstructive pulmonary disease. Am J Respir Crit Care Med 1998; 157: 1877-1880.

43 Allard P, Lamontagne C, Bernard P, et al. How effective are supplementary doses of opioids for dyspnea in terminally ill cancer patients? A randomized continuous sequential clinical trial. J Pain Symptom Manage 1999; 17: $256-265$.

44 Beauford W, Saylor TT, Stansbury DW, et al. Effects of nebulized morphine sulfate on the exercise tolerance of the ventilatory limited COPD patient. Chest 1993; 104: 175-178.

45 Gamborg H, Riis J, Christrup L, et al. Effect of intraoral and subcutaneous morphine on dyspnea at rest in terminal patients with primary lung cancer or lung metastases. J Opioid Manag 2013; 9: 269-274.

46 Grimbert D, Lubin $\mathrm{O}$, de Monte $\mathrm{M}$, et al. Dyspnée et aérosols de morphine dans les soins palliatifs du cancer broncho-pulmonaire. [Dyspnea and morphine aerosols in the palliative care of lung cancer]. Rev Mal Respir 2004; 21: 1091-1097.

47 Johnson MJ, McDonagh TA, Harkness A, et al. Morphine for the relief of breathlessness in patients with chronic heart failure - a pilot study. Eur J Heart Fail 2002; 4: 753-756.

48 Krajnik M, Podolec Z, Siekierka M, et al. Morphine inhalation by cancer patients: a comparison of different nebulization techniques using pharmacokinetic, spirometric, and gasometric parameters. J Pain Symptom Manage 2009; 38: 747-757.

49 Masood AR, Reed JW, Thomas SH. Lack of effect of inhaled morphine on exercise-induced breathlessness in chronic obstructive pulmonary disease. Thorax 1995; 50: 629-634

50 Munck LK, Christensen CB, Pedersen L, et al. Codeine in analgesic doses does not depress respiration in patients with severe chronic obstructive lung disease. Pharmacol Toxicol 1990; 66: 335-340.

51 Natalini G, Di Maio A, Rosano A, et al. Remifentanil improves breathing pattern and reduces inspiratory workload in tachypneic patients. Respir Care 2011; 56: 827-833.

52 Navigante AH, Castro MA, Cerchietti LC. Morphine versus midazolam as upfront therapy to control dyspnea perception in cancer patients while its underlying cause is sought or treated. J Pain Symptom Manage 2010; 39: $820-830$.

53 Otulana B, Okikawa J, Linn L, et al. Safety and pharmacokinetics of inhaled morphine delivered using the AERx system in patients with moderate-to-severe asthma. Int J Clin Pharmacol Ther 2004; 42: 456-462.

54 Rice KL, Kronenberg RS, Hedemark LL, et al. Effects of chronic administration of codeine and promethazine on breathlessness and exercise tolerance in patients with chronic airflow obstruction. $\mathrm{Br} J$ Dis Chest 1987; 81: 287-292.

55 Robin ED, Burke CM. Single-patient randomized clinical trial. Opiates for intractable dyspnea. Chest 1986; 90: 888-892.

56 Schönhofer B, Köhler D. Stellenwert von oral appliziertem retardiertem Morphin zur Therapie des schwergradigen Lungenemphysems vom Pink-Puffer-Typ: Eine Pilotstudie. [Value of orally administered retard morphine for therapy of severe pulmonary emphysema of the pink-puffer type. A pilot study]. Dtsch Med Wochenschr 1998; 123: 1433-1438.

57 Smith TJ, Coyne P, French W, et al. Failure to accrue to a study of nebulized fentanyl for dyspnea: lessons learned. J Palliat Med 2009; 12: 771-772.

58 Williams SG, Wright DJ, Marshall P, et al. Safety and potential benefits of low dose diamorphine during exercise in patients with chronic heart failure. Heart 2003; 89: 1085-1086.

59 Allcroft P, Margitanovic V, Greene A, et al. The role of benzodiazepines in breathlessness: a single site, open label pilot of sustained release morphine together with clonazepam. J Palliat Med 2013; 16: 741-744.

60 Boyd KJ, Kelly M. Oral morphine as symptomatic treatment of dyspnoea in patients with advanced cancer. Palliat Med 1997; 11: 277-281.

61 Bruera E, Macmillan K, Pither J, et al. Effects of morphine on the dyspnea of terminal cancer patients. J Pain Symptom Manage 1990; 5: 341-344.

62 Clemens KE, Klaschik E. Symptomatische Therapie der Dyspnoe bei Patienten in der Palliativmedizin: Sauerstoff-Insufflation versus Opioidapplikation. [Symptomatic treatment of dyspnoea in patients receiving palliative care: nasal delivery of oxygen compared with opioid administration]. Dtsch Med Wochenschr 2007; 132: 1939-1943

63 Clemens KE, Klaschik E. Morphine in the management of dyspnoea in ALS. A pilot study. Eur J Neurol 2008; 15 445-450.

64 Clemens KE, Klaschik E. Effect of hydromorphone on ventilation in palliative care patients with dyspnea. Support Care Cancer 2008; 16: 93-99.

65 Clemens KE, Quednau I, Klaschik E. Is there a higher risk of respiratory depression in opioid-naive palliative care patients during symptomatic therapy of dyspnea with strong opioids? J Palliat Med 2008; 11: 204-216.

66 Clemens KE, Quednau I, Klaschik E. Use of oxygen and opioids in the palliation of dyspnoea in hypoxic and non-hypoxic palliative care patients: a prospective study. Support Care Cancer 2009; 17: 367-377.

67 Clemens KE, Klaschik E. Dyspnoea associated with anxiety - symptomatic therapy with opioids in combination with lorazepam and its effect on ventilation in palliative care patients. Support Care Cancer 2011; 19: 2027-2033. 
68 Cohen $\mathrm{MH}$, Anderson AJ, Krasnow SH, et al. Continuous intravenous infusion of morphine for severe dyspnea. South Med J 1991; 84: 229-234.

69 Coyne PJ, Viswanathan R, Smith TJ. Nebulized fentanyl citrate improves patients' perception of breathing, respiratory rate, and oxygen saturation in dyspnea. J Pain Symptom Manage 2002; 23: 157-160.

70 Currow DC, McDonald C, Oaten S, et al. Once-daily opioids for chronic dyspnea: a dose increment and pharmacovigilance study. J Pain Symptom Manage 2011; 42: 388-399.

71 Gauna AA, Kang SK, Triano ML, et al. Oral transmucosal fentanyl citrate for dyspnea in terminally ill patients: an observational case series. J Palliat Med 2008; 11: 643-648.

72 Tanaka K, Shima Y, Kakinuma R, et al. Effect of nebulized morphine in cancer patients with dyspnea: a pilot study. Jpn J Clin Oncol 1999; 29: 600-603.

73 Farncombe M, Chater S, Gillin A. The use of nebulized opioids for breathlessness: a chart review. Palliat Med 1994; 8: 306-312.

74 Farncombe M, Chater S. Case studies outlining use of nebulized morphine for patients with end-stage chronic lung and cardiac disease. J Pain Symptom Manage 1993; 8: 221-225.

75 Allen S, Raut S, Woollard J, et al. Low dose diamorphine reduces breathlessness without causing a fall in oxygen saturation in elderly patients with end-stage idiopathic pulmonary fibrosis. Palliat Med 2005; 19: 128-130.

76 Oxberry SG, Bland JM, Clark AL, et al. Repeat dose opioids may be effective for breathlessness in chronic heart failure if given for long enough. J Palliat Med 2013; 16: 250-255.

77 Sporer KA, Tabas JA, Tam RK, et al. Do medications affect vital signs in the prehospital treatment of acute decompensated heart failure? Prehosp Emerg Care 2006; 10: 41-45.

78 Benitez-Rosario MA, Martin AS, Feria M. Oral transmucosal fentanyl citrate in the management of dyspnea crises in cancer patients. J Pain Symptom Manage 2005; 30: 395-397.

79 Sitte T, Bausewein C. Intranasal fentanyl for episodic breathlessness. J Pain Symptom Manage 2008; 36: e3-e6.

80 Pang GS, Qu LM, Tan YY, et al. Intravenous fentanyl for dyspnea at the end of life: lessons for future research in dyspnea. Am J Hosp Palliat Care 2016; 33: 222-227.

81 Farncombe M, Chater S. Clinical application of nebulized opioids for treatment of dyspnoea in patients with malignant disease. Support Care Cancer 1994; 2: 184-187.

82 Colman R, Singer LG, Barua R, et al. Outcomes of lung transplant candidates referred for co-management by palliative care: a retrospective case series. Palliat Med 2015; 29: 429-435.

83 Kanemoto K, Satoh H, Kagohashi K, et al. Psychotropic drugs for terminally ill patients with respiratory disease. Tuberk Toraks 2007; 55: 5-10.

84 Sitte T. Nasale Anwendung von Fentanylzitrat zur Kupierung von Atemnot in der Palliativversorgung - Überblick und Fallbeispiel. [Nasal application of fentanyl citrate as symptom control against breathlessness in palliative care - overview and case report]. Wien Med Wochenschr 2009; 159: 566-570.

85 Verberkt CA, van den Beuken-van Everdingen MH, Franssen FM, et al. A randomized controlled trial on the benefits and respiratory adverse effects of morphine for refractory dyspnea in patients with COPD: protocol of the MORDYC study. Contemp Clin Trials 2016; 47: 228-234.

86 López-Saca JM, Centeno C. Opioids prescription for symptoms relief and the impact on respiratory function: updated evidence. Curr Opin Support Palliat Care 2014; 8: 383-390.

87 Cabezón-Gutiérrez L, Khosravi-Shahi P, Custodio-Cabello S, et al. Opioids for management of episodic breathlessness or dyspnea in patients with advanced disease. Support Care Cancer 2016; 24: 4045-4055.

88 Pauwels RA, Buist AS, Calverley PM, et al. Global strategy for the diagnosis, management, and prevention of chronic obstructive pulmonary disease. NHLBI/WHO Global Initiative for Chronic Obstructive Lung Disease (GOLD) Workshop summary. Am J Respir Crit Care Med 2001; 163: 1256-1276.

89 Sterne JA, Hernán MA, Reeves BC, et al. ROBINS-I: a tool for assessing risk of bias in non-randomised studies of interventions. BMJ 2016; 355: i4919.

90 Vozoris NT, Wang X, Fischer HD, et al. Incident opioid drug use and adverse respiratory outcomes among older adults with COPD. Eur Respir J 2016; 48: 683-693.

91 Ekström MP, Bornefalk-Hermansson A, Abernethy AP, et al. Safety of benzodiazepines and opioids in very severe respiratory disease: national prospective study. BMJ 2014; 348: g445. 